\title{
Chromatin remodelers in oligodendroglia
}

Carlos Parras ${ }^{1}$, Corentine Marie ${ }^{1}$, Chuntao Zhao ${ }^{2}$, Q. Richard Lu

${ }^{1}$ Sorbonne Université, UPMC University Paris 06, Inserm U1127, CNRS UMR 7225, GH Pitié-Salpêtrière, Institut du Cerveau et de la Moelle Épinière, ICM, 75013 Paris, France.

${ }^{2}$ Department of Pediatrics, Brain Tumor Center, Division of Experimental Hematology and Cancer Biology, Cincinnati Children's Hospital Medical Center, Cincinnati, OH 45229, USA

Running title: chromatin remodelers in oligodendroglia

\section{Main Points}

- Chromatin remodelers (CRs) regulate each stage of oligodendrogenesis

- CRs cooperate with key transcription factors to regulate (re)myelination

- CRs have distinct and overlapping functions depending on regions, processes, and genenetworks

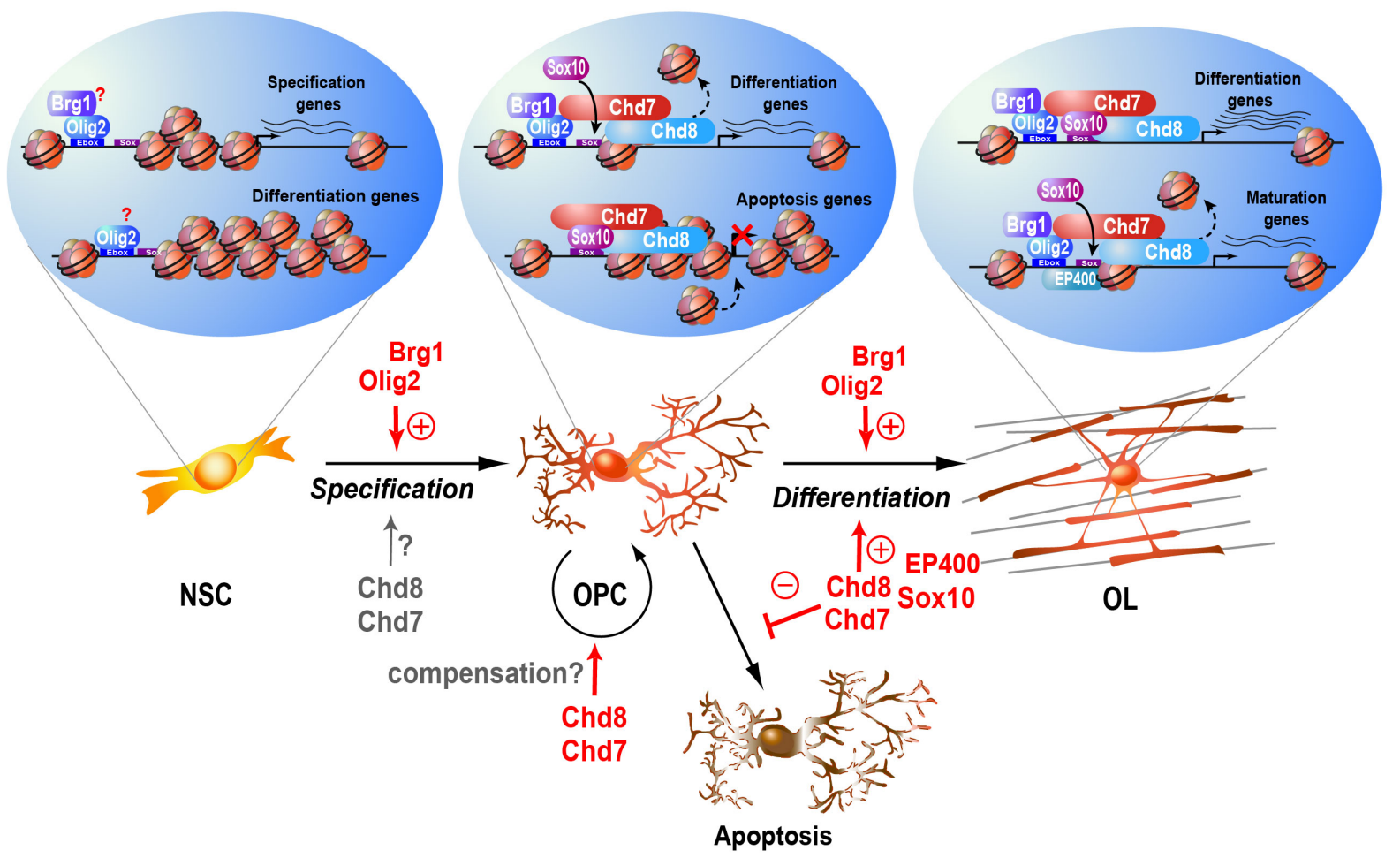

Keywords: Oligodendrogenesis; CNS development; Chromatin remodelers; Transcription regulation; Transcription factors 


\begin{abstract}
Oligodendrocytes, the myelinating cells in the vertebrate central nervous system, produce myelin sheaths to enable saltatory propagation of action potentials. The process of oligodendrocyte myelination entails a stepwise progression from precursor specification to differentiation, which is coordinated by a series of transcriptional and chromatin remodeling events. ATP-dependent chromatin remodeling enzymes, which utilize ATP as an energy source to control chromatin dynamics and regulate the accessibility of chromatin to transcriptional regulators, are critical for oligodendrocyte lineage development and regeneration. In this review, we focus on the latest insights into the spatial and temporal specificity of chromatin remodelers during oligodendrocyte development, myelinogenesis, and regeneration. We will also bring together various plausible mechanisms by which lineage specific transcriptional regulators coordinate with chromatin remodeling factors for programming genomic landscapes to specifically modulate these different processes during developmental myelination and remyelination upon injury.
\end{abstract}




\section{Introduction: Transcriptional regulation: Transcription factors and chromatin remodelers}

One of the most intriguing aspects of cell biology is how an individual cell in a multicellular organism establishes and maintains its own personalized genetic program, given that all cell-subtypes have the same genetic information. In all developing organisms, stem/progenitor cells give rise to more specialized cells over time. For instance, embryonic stem cells (ESCs) generate neural stem cells (NSCs), some NSCs then produce oligodendrocyte precursor cells (OPCs) which in turn differentiate into immature oligodendrocytes (OLs) and eventually give rise to mature myelinating OLs. Each of these cells has different morphologies and functions, mainly due to the different gene-sets they express. Moreover, it is this active genetic program what makes OLs different from neurons or astrocytes despite all three sharing a common pool of NSCs. These genetic programs and differentiation processes have been revealed by the recent advances in transcriptomic technologies, allowing us to compare the different genetic programs in detail. How transcriptional programs are established and regulated in the context of $\mathrm{OL}$ lineage development is the main topic of this review, with a focus on the role that chromatin remodelers play together with transcription factors.

\subsection{Transcriptional machinery for oligodendrocyte development}

Gene expression is mainly regulated by cis-regulatory regions which are called promoters, if they are placed at the start of the gene locus, or enhancers, if they are positioned relatively distal to the start site. The concerted activity of promoters and enhancers is responsible for ensuring proper spatiotemporal expression patterns of eukaryotic genes (Kim \& Shiekhattar, 2015). Direct binding of enhancers to cognate promoters is mediated by different types of proteins including transcription factors (TFs), cofactors, the mediator complex, and remodeling factors (Deng et al., 2014). TFs recognize specific DNA sequences and thus regulate the transcriptional activity of target genes. They are classified by their DNA binding domains into different families such as the basic helix-loop-helix (bHLH; (Bertrand, Castro, \& Guillemot, 2002) like Olig2 and Ascl1, the high mobility group-box (HMG; (Malarkey \& Churchill, 2012) such as Sox10, or the homeodomain family such as Nkx2.2. These domains usually bind to specific DNA sequences present in regulatory elements consisting of 4-10 nucleotide sequences, called TF binding motifs. The role of TFs is to recruit the basal transcription machinery that will link the enhancers to the promoter and allow robust transcription (Panne, 2008). For instance, Sox10 can directly interact with the mediator complex in order to recruit the RNA polymerase II to drive transcription (Vogl et al., 2013).

Different mechanisms leading to gene repression have been described, including recruitment of histone deacetylases (HDACs) (Ye et al., 2009), regulating chromatin structure (Chen, Fernandez, Mische, \& Courey, 1999), directly binding to histones preventing activator recruitment (Sekiya \& Zaret, 
2007), or by binding to and blocking TF sites (Y. Cai, Brophy, Levitan, Stifani, \& Dressler, 2003). For instance, repressive TFs, such as Hes and ID proteins, have also been involved in OPC cell fates. On the one hand, Hes bHLH proteins (Hairy/Enhancer of split), canonical targets of Notch signaling (Akazawa, Sasai, Nakanishi, \& Kageyama, 1992), can recruit corepressors, such as Groucho/TLE (transducin-like enhancer of split), or histone deacetylases, to inhibit gene transcription (Kageyama, Ohtsuka, \& Kobayashi, 2007), and in OPCs, Hes proteins can bind and sequester other TFs, such as Ascl1, Olig2 and Sox10 (Kageyama et al., 2007; A. Liu et al., 2006). On the other hand, ID (inhibitors of DNA-binding) $\mathrm{HLH}$ proteins are able to form heterodimers with other bHLH proteins but lacking the DNA-binding basic domain are unable to bind DNA (Benezra, Davis, Lockshon, Turner, \& Weintraub, 1990; Norton, Deed, Craggs, \& Sablitzky, 1998), with Id2 and Id4 playing an inhibitory role in the timing of OL differentiation (Kondo \& Raff, 2000; S. Wang, Sdrulla, Johnson, Yokota, \& Barres, 2001).

\subsection{Pioneer TFs and recruitment of chromatin remodelers}

The local chromatin compaction status is structurally and intrinsically repressive because nucleosomes spaced along DNA can hide the binding site of TFs, preventing unwelcomed gene expression (Zaret \& Mango, 2016). Modifications of histones composing the nucleosome (H2A, H2B, H3, and $\mathrm{H} 4$ ) can alter gene transcription by physical obstruction, reducing chromatin availability to TFs, or by posttranslational modifications influencing chromatin compaction and accessibility (Lawrence, Daujat, \& Schneider, 2016). Some histone modifications are associated with transcription activation such as trimethylation of lysine 4 in histone 3 (H3K4me3) in promoters (Barski et al., 2007; Guenther, Levine, Boyer, Jaenisch, \& Young, 2007) and mono- or di-methylation (H3K4me1/2) in enhancers. In addition to $\mathrm{H} 3 \mathrm{~K} 4 \mathrm{me} 1 / 3$, mutually exclusive modifications on H3K27 residues co-segregate with active or inactive/poised enhancers (Creyghton et al., 2010; Rada-Iglesias et al., 2011). Active regulatory elements are enriched in the H3K27ac mark (Z. Wang et al., 2008), a major substrate for the histone acetyltransferase p300/CBP (Jin et al., 2011), while inactive regulatory elements are marked by H3K27me3, deposited by the histone methyltransferase PCR2 complex (Rada-Iglesias et al., 2011).

To overcome these physical barriers, a set of TFs, called pioneer TFs, acts as transcription initiators (Cirillo et al., 2002). They can bind to DNA in the context of nucleosomes (Cirillo et al., 2002; Cirillo et al., 1998), often having a reduced binding motif that can be exposed at the surface of the nucleosome (Soufi et al., 2015). This initial binding allows the recruitment of other factors that can in turn change the local chromatin structure (Zaret \& Mango, 2016). For example, Ascl1, a key proneural TF, has been shown to behave as a pioneer TF in NPCs (Raposo et al., 2015), in line with its capacity to rearrange the chromatin and induce neuronal-reprogramming in different cell types, including fibroblasts (Wapinski et al., 2013). Pioneer TFs can attract chromatin-modifying factors that will alter the chromatin by different mechanisms, such as DNA methylations, histone modifications and ATP- 
dependent chromatin alterations. Interestingly, an early study highlighted a large chromatin reorganization during OPC differentiation into OLs (Nielsen, Hudson, \& Armstrong, 2002), underscoring the importance of these remodeling mechanisms in oligodendroglial lineage progression. Here, we will focus on ATP-dependent chromatin remodelers which can (re)move nucleosomes and thus modify chromatin accessibility.

\subsection{Chromatin remodeler families and their roles during CNS development}

ATP-dependent chromatin remodelers use the energy from ATP hydrolysis to modify chromatin structure, dynamically altering the local nucleosomes as these remodelers can translocate, evict, add, or change histones on the DNA (Woodage, Basrai, Baxevanis, Hieter, \& Collins, 1997). These chromatin remodelers are part of the SF2 superfamily as they present a common helicase ATPase domain. They are subdivided into four major subfamilies depending on their other domains: i) the Switch/Sucrose Non-Fermentable (SWI/SNF) complex, contain either Brahma (Brm or Smarca2) or Brahma-related gene 1 (Brg1 or Smarca4) ATP subunits, two homolog factors also having a bromodomain that binds to acetylated histones (Ferri, Petosa, \& McKenna, 2016); ii) the Imitation SWI (ISWI) complex is characterized by the presence of a HAND-SANT-SLIDE domain which binds to nucleosomes and nucleosomal DNA. It is composed of SNF2H (Smarca5), which is essential for cell survival (AlvarezSaavedra et al., 2014), or SNF2L (Smarca1), which participates in brain development (Yip et al., 2012); iii) the Inositol-auxotroph-mutant-80/SWI/SNF-related (INO80/SWR) complex can deposit or remove histones subtypes such as H2A.Z (Yen, Vinayachandran, \& Pugh, 2013); and iv) chromodomain helicase DNA binding (CHD) proteins, comprising nine different members (CHD1-9), characterized by the presence of two chromodomains shown to bind to methylated histones.

\section{Chromatin remodelers and oligodendroglia}

Oligodendrogenesis is a complex process which can be split in three different steps (Fig. 1A): First, the specification phase corresponding to NSCs commitment to the oligodendroglial lineage, initially as intermediate primitive OPCs (pri-OPCs or pre-OPCs) (Filbin \& Monje, 2019; Weng et al., 2019) that then generate the OPCs, a stable precursor with an extended proliferative capacity (Dawson, Polito, Levine, \& Reynolds, 2003). Second, the phase of OPC differentiation into postmitotic immature oligodendrocytes (iOLs) with their typical elaborated stellar morphology. Finally, the maturation of iOLs into the myelinating oligodendrocytes (mOLs) involving the process of enwrapping and myelination of multiple axonal segments. Interestingly, compaction of the chromatin increases during differentiation (Nielsen et al., 2002), correlating with the upregulation of chromatin-remodeling genes in the transition from OPCs to iOLs (Fig. 1A,B), as shown by the strong binding of the RNA polymerase 
II to their gene promoters (Yu et al., 2013). These data suggest that oligodendroglia differentiation involves extensive chromatin remodeling and reorganization of the transcriptional machinery.

In 2000, Olig1 and Olig2 bHLH TFs were identified as key regulators of oligodendrogenesis (Lu et al., 2000; Qiao Zhou, Wang, \& Anderson, 2000) and subsequently shown to be required and, in certain cellular contexts, sufficient to drive differentiation of NSCs into oligodendroglia (Z. Liu et al., 2007; Lu et al., 2002; Q. Zhou \& Anderson, 2002). Olig2 has a predominant role, given that in Olig2 knockout mice, OPCs are never formed throughout the CNS, with the exception of a few hindbrain OPCs depending on Olig1 for their generation (Lu et al., 2002; Q. Zhou \& Anderson, 2002). Furthermore, Olig2 forebrain-specific deletion causes severe deficits in myelination at postnatal stages (Yue et al., 2006), while in vivo Olig2 overexpression increases OL numbers and induces precocious myelination (Wegener et al., 2015). Mechanistically, and contrary to its initially proposed role as a repressor (Marshall, Novitch, \& Goldman, 2005; Novitch, Chen, \& Jessell, 2001), Olig2 has been later shown to act as a transcription activator in oligodendroglia forming heterodimers with E47 E-protein (Kuspert, Hammer, Bosl, \& Wegner, 2011), and that Olig2 binding is required for the activation of many genes involved in oligodendrogenesis (Yu et al., 2013), including Sox10, another key TF in this process (Stolt et al., 2002; Turnescu et al., 2018). Interestingly, the five chromatin remodelers (i.e. Brg1, Brm, Chd7, Chd8, and EP400) already shown to play important roles in oligodendrogenesis, are Olig2 and Sox10 target genes, and cooperate with Olig2 and Sox10 in some of these functions (Bischof, Weider, Küspert, Nave, \& Wegner, 2015; He et al., 2016; Marie et al., 2018; Matsumoto et al., 2016; Yu et al., 2013). We detail below our present understanding of their implications in different processes of oligodendrogenesis starting from best-characterized ones.

\subsection{OPC differentiation and myelination}

\subsubsection{Brg1 is required for OPC differentiation}

Brg1 was the first remodeler implicated in oligodendrogenesis. It was shown to be a direct target of Olig2, given that Olig2 binds the gene regulatory regions of Brg1 in OPCs and iOLs, and Brg1 is downregulated in Olig2 conditional knockout (hGFAP-Cre driver) (Yu et al., 2013). Interestingly, Brg1 deletion mediated by Olig1-Cre, which induces recombination in NSCs of the spinal cord motor neuron domain (pMN) and in OPCs elsewhere in the CNS (Lu et al., 2000; Xin et al., 2005; Qiao Zhou et al., 2000), presents normal numbers of embryonic and postnatal OPCs, but mature OLs are depleted and mice lack myelination, leading to tremors, limb paralysis, and death before the end of the first postnatal month (Yu et al., 2013). Another study deleting Brg1 using CNP-Cre (that recombines in 80$90 \%$ of OPCs during embryogenesis) or NG2-Cre (exhibiting lower recombination rates in OPCs: $30 \%$ at late embryogenesis and $\sim 50 \%$ at postnatal stages) drivers, also found no reduction in Brg1-deleted 
OPC numbers in the spinal cord (Bischof et al., 2015), but only found a partial reduction ( $40 \%$ ) in OLs generated from Brg1-deleted OPCs. The different phenotypes found between Olig1-Cre- and NG2-Credriven Brg1-deletion could be due to the timing of Brg1 deletion in OPCs, given that Olig1 expression precedes NG2 and CNP. It is possible that Brm, an alternative ATPase-subunit of the SWI/SNF/BAF complexes, may compensate Brg1 functions in BAF complexes (Hargreaves \& Crabtree, 2011; L. Ho \& Crabtree, 2010). However, despite the fact that Brm and Brg1 are largely coexpressed during oligodendroglia, Brm knockdown and chromatin immunoprecipitation (ChIP) experiments suggest that this may not be the case (Bischof et al., 2015) and that Brg1 is largely indispensable for OPC differentiation (Figure 2).

\subsection{2 $\mathrm{Brg} 1$ is recruited by Olig2 to activate oligodendroglial gene transcription}

In Schwann lineage cells, Brg1 binds Sox10 regulatory elements and Sox10 is essential for Brg1 chromatin recruitment to activate the differentiation (Weider et al., 2012). On the contrary, in OLs, no evidence was found for a similar relationship between both proteins, despite that they bind to each other in these cells (Bischof et al., 2015). The specific expression of Sox10 paralogs, Sox8 and Sox9, and Olig1/2 TFs, in OL lineage cells but not in Schwann cells, could account for these differences. Indeed, Brg1 can bind the Olig2 protein (Yu et al., 2013). Interestingly, the majority of Brg1-bound regions in iOLs are already bound by Olig2 in OPCs (Yu et al., 2013). Therefore, a model has been proposed (Yu et al., 2013) suggesting that Olig2, but not Sox10, acts as a pioneer TF in OPCs to recruit Brg1 to its binding sites and thus remodel chromatin to induce robust gene expression of OPC differentiation and myelination genes (Figure 2).

\subsubsection{Chd7 is required for normal CNS myelination}

Brg1 and Olig2 chromatin binding profiles (by ChIP-seq, ChIP followed by DNA sequencing) in iOLs indicate their co-occupancy in active regulatory regions of the Chd7 locus (He et al., 2016), suggesting that Chd7 chromatin remodeler is a direct target of Brg1 and Olig2. Indeed, Chd7 transcripts are downregulated upon Olig1-Cre mediated Brg1 deletion (He et al., 2016), and thus in Olig1-Cre mediated Olig2 deletion, where Brg1 is downregulated (Yu et al., 2013). Chd7 is enriched in OL lineage cells, with Chd7 protein levels peaking in iOLs and decreased in mOLs (Figure 1), but mostly absent from neurons, astrocytes, and microglia in the postnatal brain (He et al., 2016; Marie et al., 2018). This enrichment in iOLs, points towards a role for Chd7 in OL differentiation and/or myelination. Indeed, mice with Olig1-Cre mediated Chd7-deletion, despite having normal numbers of OPCs, have a 50\% reduction in OLs both the brain and spinal cord by the second postnatal week (He et al., 2016). Similarly, reduced OL differentiation and myelination was found in the brain after an acute Chd7deletion in neonatal OPCs (using PDGFRa-CreERT driver). Remarkably, these defects were transient and eventually OL numbers and myelination catch up at adult stages, suggesting that Chd7 is not fully 
required for normal OL differentiation and myelination, but it is involved in the onset of myelination (He et al., 2016).

\subsubsection{Chd7 function is required to induce OPC differentiation but not OL stage progression}

The stage and extent of Chd7 requirement in OL generation, was addressed using a neonatal OPCspecific Chd7 deletion (Chd7iKO mice using PDGFRa-CreERT driver), leading to a decrease ( $40 \%)$ in Nkx2.2 ${ }^{+}$iOLs compared to control brains, indicating that Chd7 deletion in OPCs indeed impairs normal OPC differentiation (Marie et al., 2018). Furthermore, using several markers labeling subsets of differentiating OLs, such as Itpr2 (Marques et al., 2016), APC (Lang et al., 2013), or different levels of CC1 \& Olig1 (Nakatani et al., 2013), a similar reduction of each OL subset was found in Chd7iKO brains (Marie et al., 2018), indicating that Chd7 loss reduces the number of differentiating OLs but does not alter OL stage progression once OPC differentiation is started. Altogether, these data indicate that Chd7 deletion only partially impairs OPC capacity to start differentiation (Figure 2), suggesting that other chromatin remodeling factors may play redundant functions with Chd7 in OPC differentiation process.

\subsubsection{Chd7 interacts with Sox10 to regulate the myelinogenic program}

Chd7 chromatin binding profiling in differentiating OLs shows Chd7 binding to the regulatory regions of many genes encoding key regulators of myelinogenesis (including Sox10, Myrf, Olig1, Zfp191, and Sip1/Zeb2), lipid metabolism, cytoskeleton assembly, and myelin components (such as Mbp, Ugt8, Plp1, and Cnp) (He et al., 2016). Interestingly, analysis of the DNA sequence motifs enriched in Chd7 bound regions identify Sox10 consensus binding motif as the best matching binding site. Given that CHD remodelers bind chromatin through binding to TFs, Sox10 could function as a recruiting factor to bring Chd7 to its binding sites, and thus induce remodeling of these regulatory regions. In support of this hypothesis, Sox10 can directly bind Chd7 in oligodendroglial cells (He et al., 2016), Sox10 chromatin binding peaks overlap with $40 \%$ of Chd7 peaks in OLs, and the height of Sox 10 binding peaks is higher when Chd7 is also present at those peaks (He et al., 2016), and they present higher signal of H3K27Ac active transcription histone mark, suggesting that the Chd7 and Sox10 co-binding could promote gene activation (Figure 2). Indeed, the cooperation of Sox10 and Chd7 to activate genes involved in OL differentiation and myelination is supported by gain-of-function experiments in cultured cells showing that Chd7 can increase the transcription activity of Sox10 on myelin-protein genes (He et al., 2016). Together these data suggest that Sox10 and Chd7 are part of a physical complex targeting chromatin regulatory regions (Figure 2), and that Chd7 binding reinforces Sox10 binding and its transcription activity at their commonly bound sites.

\subsubsection{Chd8 is strongly required for OPC differentiation}


Despite Chd7 binding to many genes related to OPC differentiation, only a fraction of these genes are deregulated upon Chd7 deletion, and the process is only partly defective, suggesting a partial requirement of Chd7 in OPC differentiation (He et al., 2016; Marie et al., 2018). Interestingly, Chd8, the Chd7 paralogue, presents a similar expression pattern to Chd7 in oligodendroglia, with Chd8 protein present in OPCs, having its highest levels in iOLs, and decreasing to almost undetectable levels in mOLs (Marie et al., 2018; Zhao et al., 2018), in agreement with Chd8 transcripts in postnatal brain as reported by RNA-seq transcriptomes (Figure 1) (Marques et al., 2018; Marques et al., 2016; Zhang et al., 2014)).

To investigate Chd8 function in oligodendrogenesis, Chd8 has been deleted using the Olig1Cre driver (Chd8cKO), and similar to Brg1cKO mice, Chd8cKO mice presents a severe dysmyelination, seizures by P14, and death by P21 (Zhao et al., 2018). Remarkably, oligodendroglial cells (Mbp ${ }^{+}$and $P / p 1^{+}$cells) are completely absent in both the spinal cord and cerebellum at P14, but only partially reduced in the forebrain. The reduced OL generation in Chd8cKO also leads to a lack of axonal myelination in the optic nerve, spinal cord, and to a lesser extent in the corpus callosum (Zhao et al., 2018), suggesting that the extent of Chd8 requirement for OL generation and myelination depends on the CNS region. Confirming the requirement of Chd8 in OL differentiation, another study performing Chd8 knockdown in neonatal brain-derived NSC differentiating cultures found reduced (by $36 \%$ ) OL differentiation without changes in OPC numbers in Chd8 knockdown cells compared to controls (Marie et al., 2018). Together, these observations indicate a spatiotemporal specific role of Chd8 in regulating CNS myelination.

\subsubsection{Chd8 establishes open chromatin at promoters to activate OL differentiation genes}

Supporting that Chd8 directly activates the transcription programs mediating OL differentiation and myelination, transcriptome comparison between Chd8cKO and control OPCs showed downregulation of key regulators (including Olig1, Olig2, Sox10, and Myrf) and myelin protein genes. Furthermore, Chd8 chromatin binding profiling in OPCs (by ChIP-seq) indicates that Chd8 mostly binds promoter regions also bound by Olig2 and Sox10, and Chd8-occupied genes deregulated in Chd8-deleted OPCs are enriched in OL differentiation GO terms (Zhao et al., 2018), suggesting that Chd8 directly regulates genes involved in $\mathrm{OL}$ differentiation. To assess whether Chd8 directly promotes transcriptional activation by facilitating nucleosome depletion at promoters of these genes, accessible chromatin profiling of OPCs isolated from Chd8cKO and control brains have been compared, using ATAC-seq (Assay for Transposase-Accessible Chromatin followed by DNA sequencing). Interestingly, chromatin accessibility in the promoters of OL differentiation-related genes were drastically decreased in Chd8cKO OPCs (Zhao et al., 2018). Therefore, these data suggest that Chd8 induces chromatin accessibility at gene promoters in order to activate the OPC differentiation program (Figure 2). 
Interestingly, in Chd8-deficient cells, genes marked by H3K4me3 (mark of promoter activity) and bound by histone methyltransferase KMT2/MLL (that deposits H3K4me3 mark) are downregulated, suggesting that Chd8 promotes a transcriptional initiation program for OPC differentiation by recruiting $\mathrm{KMT2/MLL}$ activity.

\subsubsection{Chd8 compensates for Chd7 LOF during OPC differentiation}

To investigate whether Chd7 and Chd8 regulate common OPC genetic programs, Chd7 and Chd8 chromatin binding profiles from in vivo purified OPCs were compared, and interestingly, it was found that the large majority (84\%) of genes bound by Chd7 at their promoter and enhancer regions are also bound by Chd8, including genes well-known regulators of OL differentiation (such as Olig2, Olig1, Ascl1, Nkx2.2, and Sox10) (Marie et al., 2018). Moreover, direct evidence that Chd7 and Chd8 play overlapping roles in OL differentiation was obtained using NSC differentiating cultures with different gene-dose reductions of $C h d 7$ and $C h d 8$, showing that while the loss of one Chd7 allele did not affect OPC differentiation, its combination with Chd8-knockdown further reduced the ratio between iOLs and OPCs compared to Chd8-knockdown alone (Marie et al., 2018). These results indicate that Chd7 and Chd8 genetically interact in the process of OPC differentiation, and given their common target genes, suggest that Chd8 may partially compensate the loss of Chd7 during OPC differentiation (Figure 2). The question as to whether or not removing both Chd7 and Chd8 completely impairs OL differentiation awaits further studies.

\subsubsection{EP400 is required for Myrf activation and OL differentiation}

The role of INO80/SWR chromatin remodelers have been recently studied in oligodendroglia deleting Ep400, the central ATP-hydrolyzing subunit of the TIP60/EP400 complex, at defined times of mouse OL development (Elsesser et al., 2019). Interestingly, upon Ep400 deletion in NSCs (Nestin-Cre driver), Ep400-deficient OPCs develop normally but terminal OL differentiation and myelination are strongly impaired both in the spinal cord and the forebrain (Elsesser et al., 2019). Mechanistically, Ep400 interacts with Sox10, both factors bind together to Myrf gene regulatory regions, and are required for Myrf expression (Elsesser et al., 2019), a key TF promoting OL maturation and myelination programs (Emery et al., 2009). In OPCs, Ep400 seems to precede Sox10 in its binding to Myrf regulatory regions, therefore, other factors than Sox10 might lead to Ep400 specific chromatin binding profile in oligodendroglia. Many Ep400-deleted OLs present increased DNA damage and die by apoptosis, leading to a strong reduction in myelination. On the contrary, Ep400 specific deletion in mature OLs do not present apparent defects suggesting that Ep400 is dispensable for myelin maintenance (Elsesser et al., 2019). Therefore, this study indicates that INO80/SWR chromatin remodeling complex containing EP400 is dispensable for OPC specification and mature OL maintenance but largely required for OL terminal differentiation at least in part by its cooperation with Sox10 to induce Myrf gene 
expression. Comparing genes deregulated in Ep400-deleted oligodendroglia with genes deregulated by Chd7 or Brg1 deletion showed that a $15-20 \%$ of genes were similarly dysregulated without pointing to OL gene networks specifically requiring EP400 activity (Elsesser et al., 2019).

Altogether, these studies involving five different chromatin remodelers in OL differentiation, indicate that the expression of genes regulating this process requires one or more chromatin remodelers, and that our present understanding of their implication do not allow us to assign a single remodeler to a single aspect of OL differentiation or gene-networks, but showing clear overlaps in the specific functions they have acquired during evolution.

\subsection{OPC proliferation}

OPCs are the main proliferative cells of the brain (Dawson et al., 2003), with OPCs labeled embryonically generating clones of up to 400 cells (García-Marqués, Núñez-Llaves, \& LópezMascaraque, 2014). Furthermore, OPCs seems to be the main amplifying-cell involved in adult gliomas (C. Liu et al., 2011). Therefore, the regulation of OPC proliferation has to be tightly controlled, including by chromatin remodelers.

\subsubsection{Chd7 is largely dispensable for brain OPC proliferation}

Brains where Chd7 is specifically deleted in OPCs (Chd7iKO, PDGFRa-CreERT driver) at neonatal stages, present no changes in cell cycle length or numbers of proliferating OPCs when analyzed at P7 and P20 (Marie et al., 2018). Despite this observation, the transcriptome comparison (RNA-seq) between these OPCs purified from P7 brains showed that many genes involved in cell cycle regulation (such as Ccnd1, Cdk4, and Cdk6) are bound by Chd7 and upregulated in Chd7-deleted OPCs (Marie et al., 2018). This paradox was explained by the specific reduction of the number of non-proliferative OPCs found in Chd7iKO brains, that leads to an increased fraction of proliferating OPCs in Chd7iKO brains, and accounts for the larger representation of proliferation-related transcripts found in Chd7iKO-OPC transcriptomes. Therefore, Chd7-deficient brain OPCs are not impacted in their proliferation or cell cycle length despite Chd7 binding to regulatory regions of many cell cycle related genes (Marie et al., 2018), suggesting compensation by other factor(s).

In contrast, another study focused in the spinal cord, using the same genetic tools, reported reduced OPC proliferation of Chd7iKO mice compared to controls, by 3-fold at embryonic day 13 and 2 -fold in adult mice (Doi et al., 2017). With respect to Chd7 requirement for brain OPC proliferation (He et al., 2016; Marie et al., 2018), given the lack of analysis in this region by Doi and collaborators, the differences between these studies could be explained by region-specific differences in Chd7 requirement between the brain and spinal cord. However, concerning the spinal cord, the results on OPC proliferation in embryonic or adult Chd7-deleted OPCs (Doi et al., 2017) differ from those using 
Olig1-Cre to induce Chd7 deletion, which showed comparable OPC numbers in Chd7 mutant and control spinal cords at postnatal stages (P0, P7 and P21), and similar proportion of proliferating OPCs at P7 (He et al., 2016). Thus, further studies are required to solve this discrepancy.

\subsubsection{Chd8 is partially required for OPC proliferation}

In contrast to Chd7-deletion, Olig1-Cre mediated deletion of Chd8 drastically decreased the number of OPCs, and their proliferation in the developing spinal cord, but in the corpus callosum of the same animals, no reduction was found neither in OPC numbers, nor in their proliferation (Zhao et al., 2018). These regional differences in the numbers of OPCs present in Olig1-Cre/Chd $8^{f / f l}$ mice could be due to different requirements of Chd8 in oligodendrogenesis in the forebrain and spinal cord, or due to differences in the timing/extent of Chd8 deletion in each region. Supporting this second hypothesis, deleting Chd8 in neonatal OPCs (using the PDGFR $\alpha$-CreERT driver, Chd8iKO), led to a strong (>85\%) OL reduction in both the cortex and the spinal cord of Chd8iKO mice at P14, without changes in OPC proliferation and numbers, in neither the spinal cord nor the cortex (Zhao et al., 2018). Together, these results, using two different drivers to delete Chd8 at different stages of oligodendroglial lineage cells, indicate that Chd8 is only critical for initial proliferation of OPCs in the embryonic spinal cord, but later, similar to Chd7, normal OPC proliferation occurs without Chd8 both in the spinal cord and cortex (Zhao et al., 2018), suggesting that Chd8 is dispensable for proliferation of preexisting OPCs in the postnatal CNS.

Altogether, this data, including the lack of Brg1 requirement in OPC proliferation (Yu et al., 2013), indicates that $\mathrm{Chd7}$, Chd8, and Brg1, could play partially redundant functions to ensure normal OPC proliferation (Figure 2).

\subsection{OPC survival}

\subsubsection{Chd7 protects OPC from apoptosis by Trp53 direct repression}

The tumor suppressor p53, a 53kDa protein encoded by $\operatorname{Trp} 53$ gene, is well known by its capacity to promote apoptosis, with Trp53 inactivating mutations common in human tumors and linked to poor patient prognosis (Kastenhuber \& Lowe, 2017). Interestingly, Trp53 heterozygosity has been recently shown to partially rescue CHARGE-related phenotypes of Chd7-null mouse embryos, and that p53 inappropriate activation leads to CHARGE syndrome traits (Van Nostrand et al., 2014). In the brain of Chd7iKO mice, non-proliferative Chd7-deficient OPCs are reduced in numbers, suggesting that Chd7 may regulate OPC survival (Marie et al., 2018). Supporting this possibility, genes bound by Chd7 and upregulated in Chd7iKO OPCs are enriched in apoptosis/survival GO categories, including key regulators of apoptosis, such as p53/Tpr53, Bax, and Apaf1 (Marie et al., 2018). Indeed, apoptotic OPCs 
are observed in Chd7iKO brains at P7 but rarely in controls, and p53 protein is upregulated specifically in Chd7iKO non-cycling OPCs, suggesting that loss of Chd7 may result in specific apoptosis of noncycling OPCs mediated by p53 upregulation. A causal link between p53 upregulation and apoptosis in Chd7-deleted OPCs was demonstrated using a p53 specific inhibitor, Pifithrin- $\alpha$, which rescued the survival of Chd7-deleted OPCs, and restored normal OPC numbers in Chd7iKO brains (Marie et al., 2018). Furthermore, this treatment also rescued the balance between cyclin and non-cycling OPCs (40\% and 60\% respectively) in Chd7iKO brains, providing further evidence that Chd7 is dispensable for brain OPC proliferation (Marie et al., 2018). Finally, the role of Chd7 in OPC survival is in agreement with a study showing that specific Chd7 deletion in cerebellar granule neuron progenitors also increases their apoptosis (Feng et al., 2017).

At the mechanistic level, comparison of the open chromatin profiling (by ATAC-seq) between Chd7iKO OPCs and controls, shows that the promoter elements bound by Chd7 in the p53/Trp53 locus present higher chromatin accessibility in Chd7iKO OPCs compared to controls, suggesting that Chd7 may normally repress $p 53 / \operatorname{Trp} 53$ transcription by binding at these regulatory elements and inducing chromatin closing, thereby contributing to the survival of postmitotic OPCs (Marie et al., 2018). Therefore, differently to Chd7-mediated chromatin opening and gene activation of genes involved in OPC differentiation, Chd7 seems to directly promote OPC survival by chromatin closing and transcriptional repression of apoptotic genes (Figure 2).

\subsubsection{Chd8 promotes OPC survival}

Several pieces of evidence have linked Chd8 function to apoptosis. First, mice lacking Chd8 die from massive apoptosis at embryonic day 7 (Nishiyama et al., 2004). Second, Chd8 forms a trimeric complex with $\mathrm{p} 53$ and histone $\mathrm{H} 1$ on chromatin that is required for inhibition of p53-dependent transactivation and apoptosis (Nishiyama et al., 2009). Third, deletion of $p 53$ ameliorated this developmental arrest of Chd8 KO mice (Nishiyama et al., 2009). In the context of oligodendrogenesis, an increase in apoptosis of Chd8iKO OPCs was found in the spinal cord, suggesting that Chd8 is also involved in OPC survival in this region (Zhao et al., 2018). Moreover, an increase in cell death was found among OPCs isolated from Chd8cKO cortices compared with controls when maintained in differentiation medium, suggesting an increase of apoptosis upon OPC differentiation (Zhao et al., 2018). Finally, the p53 pathway is upregulated in Chd8-deleted OPCs as shown by RNA-seq and a Chd8 binding profile shows enrichment in genes associated with cell death, suggesting that upregulation of p53 signaling in Chd8deficient mutants could impair OPC survival (Zhao et al., 2018).

Together, these data show that Chd7 and Chd8 are both required in the survival of non-cycling OPCs, at least in part through Trp53 repression (Figure 2). Whether they compensate for each other 
loss in promoting survival of cycling OPCs, or other remodelers are involved (including Brg1, Brm or EP400) will need the use of combinatorial deletions of several chromatin remodelers.

\subsection{Remyelination}

\subsubsection{Chd7 is required for normal CNS remyelination}

In line with Chd7 requirement in OPC differentiation during developmental myelination, in the context of adult focal demyelination (LPC induced) of either the corpus callosum or the spinal cord, myelin repair took few days longer in Chd7-deleted mice (Olig1-Cre) than in controls, due to reduced numbers of new generated OLs (He et al., 2016), indicating that Chd7 is partially, but not completely, required for remyelination, similar to its requirement during developmental myelination (Figure 2).

\subsubsection{Chd8 is required for OPC differentiation during remyelination}

Adult Chd8iKO mice subjected to spinal cord focal demyelination, presented a reduced number of remyelinating OLs and proliferating OPCs in the lesion territory, both at 14 and 28 days post-lesion when compared to control littermates, and ultrastructure analysis at 14 days post-lesion indicates a drop from $60 \%$ to $5 \%$ of remyelinated axons in the lesion territory of Chd8iKO compared to control littermates (Zhao et al., 2018). Therefore, in the adult injured spinal cord, Chd8 is largely required for both OPC proliferation and differentiation into newly remyelinating OLs. Remarkably, together with the data from postnatal CNS, this suggests that Chd8 requirement for OPC proliferation depends on the region, developmental stage, and pathological insult. These context-dependent differences in the proliferation of Chd8-deleted OPCs may be due to compensation by Chd7 and Brg1 (Figure 2). However, Chd8 is largely required for OL differentiation, during both myelination and remyelination, either in the brain or in the spinal cord, indicating a stronger requirement of Chd8 than Chd7 in regulating $\mathrm{OL}$ differentiation process.

Therefore, both Chd7 and Chd8 are involved in normal remyelination (Figure 3), and likely play partially overlapping functions in myelin repair. Beyond $\mathrm{Chd7}$ and $\mathrm{Chd} 8$, the role of other remodeling factors in the context of myelin pathology, including Brg1 that is upregulated upon focal demyelination (Yu et al., 2013), is at present completely unexplored.

\subsection{OPC specification}

\subsubsection{Brg1 is required for OPC specification}

The expression pattern of $\mathrm{Brg} 1$ transcripts in embryonic and postnatal brain cells indicate that $\mathrm{Brg} 1$ is expressed in NPCs from embryonic stages (Figure 1) (Marques et al., 2018; Zhang et al., 2014). Remarkably, Brg1 NSC-specific deletion, driven by Nestin-Cre, causes a dramatic reduction in OPCs and OLs during embryonic CNS development, suggesting an important role of Brg1 in OPC specification 
from NSCs (Matsumoto et al., 2006). However, this phenotype is accompanied by premature NSC differentiation into neuronal fates followed by a reduced generation of astrocytes and OLs (Matsumoto et al., 2006), and due to Brg1 almost ubiquitous expression (Bischof et al., 2015), it is difficult to rule out that OPC specification defect is not secondary to NSC differentiation defects. Nevertheless, follow-up studies have shown that Olig2 ${ }^{+}$cells generated from $B r g 1$-deleted NSCs cannot become PDGFR $\alpha^{+}$OPCs or further differentiate into $\mathrm{MBP}^{+}$OLs (Nestin-Cre driver) (Matsumoto et al., 2016), or able to induce Sox10 expression (Brn4-Cre driver) (Bischof et al., 2015), supporting a requirement of Brg1 in OPC specification. Strikingly, the normal OPC numbers in Olig1-Cre/Brg1 $1^{f l f f l}$ spinal cord suggests that $\mathrm{Brg} 1$ is dispensable for OPC generation from Olig1 $1^{+}$progenitors. It might be possible that Olig1-Cre delivered an incomplete recombination in pMN NSCs for Brg1 deletion, however, this is likely not the case since Olig1-Cre-mediated Olig1/2 deletion substantially decreases OPC numbers and their proliferation (Ye et al., 2009), and no substantial differences in PDGFRa OPC numbers were found two days after the onset of OPC generation from the pMN domain (Yu et al., 2013), and before any compensation could take place from dorsally generated OPCs (J. Cai et al., 2005). Thus, certain aspects of Brg1 role in OPC specification need further investigation.

\subsubsection{Ep400 is dispensable for OPC specification}

Upon NSC-specific (Nestin-Cre driver) Ep400 deletion, Ep400-deficient OPCs develop normally but terminal OL differentiation and myelination are strongly impaired both in the spinal cord and the forebrain (Elsesser et al., 2019), thus indicating that INO80/SWR chromatin remodeling complex containing EP400 is dispensable for OPC specification.

Chd7 and Chd8 are expressed in NSCs (Feng et al., 2017; Feng et al., 2013; Marie et al., 2018; Micucci et al., 2014) (Figure 1) suggesting that they could potentially play a role in OPC specification, but this awaits for functional experimental evidence. Altogether, these data indicate that for the moment, Brg1 the only chromatin remodeler demonstrated to play an essential role in OPC specification. It remains to be determined whether Chd7 and Chd8, mutations of which lead to severe developmental disorders in humans, are also involved in OPC specification from NSCs.

\subsection{Cooperation between chromatin remodelers and TFs}

A challenge in transcription regulation is to be able to predict gene expression from the actual chromatin binding of regulatory proteins (including TFs and chromatin remodelers) in a given cell type. Integrating gene expression data from main OL lineage stages with oligodendroglia-specific genomewide chromatin profiling of chromatin remodelers (Chd7 and Chd8) and key TFs (Sox10 and Olig2), highlights a timely controlled binding of Chd7 and Chd8 to stage-specific genes in OPCs and OLs (Marie et al., 2018). This finding led us to propose a dynamic transcription regulatory model (Figure 4) (Marie 
et al., 2018) including the following steps: First, Olig2 could act as a pioneer TF, binding first to poised chromatin enhancer elements. Second, Olig2 binding would mediate the recruitment of Brg1, Chd7 and Chd8 on chromatin regulatory elements either in specific gene loci, or in a temporal sequence, or both. Third, Brg1, Chd7, and Chd8 would then induce the chromatin-opening, likely by sliding nucleosomes, thus allowing Sox10 binding to its exposed binding sites. Fourth, Sox10 activity and additional clustering of other regulators will help the mediator complex to form a stable promoterenhancer loop, and eventually, leading to the induction of robust transcription of OL differentiation genes (Figure 2). This timing of events can, not only explain the crucial importance of Olig2 and Sox10 in activating OL lineage genes (Stolt et al., 2002; Yu et al., 2013), but also suggest the partial compensation of Chd7 by Chd8 in OPC differentiation, cell survival and proliferation. Remarkably, it was found that most OPC-specific genes are still bound by the TFs in OLs, but not by chromatin remodelers (Marie et al., 2018). This could imply that the loss of Chd7 and Chd8 binding, together with the recruitment of some repressors (e.g. Nkx2.2), is required for downregulation of OPC genes and subsequent $\mathrm{OL}$ differentiation. Further studies are needed to demonstrate the predictions of this model.

\section{Conclusions}

\subsection{Specificity and redundancy between Brg1, Chd7 and Chd8}

Brg1, Chd7, Chd8, and EP400 are currently the chromatin remodelers better studied in oligodendrogenesis. They have strong to partial requirements in different stages of oligodendrogenesis (Figure 2), likely explained by partially overlapping expression patterns (Figure 1) and target genes, and thus partial redundancy in their functions. This is more apparent when comparing their differential requirements in various CNS regions, in both neurogenesis and oligodendrogenesis, and their cell- and stage-specific gene targets, suggesting that during the evolution each remodeler acquired specializations partially divergent among different neural cells and regions. Given that these remodelers do not directly recognized DNA binding motifs, their specificity relies in their association with different TFs and epigenetic marks, and mechanistic logic of their combinations is only starting to be unraveled. A summary of the different studies reviewed here are depictured in the table 1.

\subsection{Mechanisms of chromatin remodeling control of oligodendrogenesis}

Brg1 chromatin activity in oligodendroglia depends on Olig2, not only because Olig2 binds Brg1 regulatory regions and Brg1 expression is downregulated in Olig2-deleted cells, but because many Brg1-binding regions in OLs overlap with Olig2 binding in OPCs and OLs, suggesting that Brg1 binds to chromatin regions preoccupied by Olig2 in OPCs (Yu et al., 2013). Thus, a pioneer function of Olig2 has been proposed (Yu et al., 2013) to recruit Brg1 to regulatory regions still close in OPCs that will become 
open by Brg1 remodeling activity during OPC differentiation into OLs (Figure 4). This Olig2 pioneer function would be fully demonstrated by showing increase in chromatin accessibility at Olig2 bound regions during OPC differentiation and the lack of remodeling in Olig2-mutant OPCs. Chd7 expression in oligodendroglia is at least partially driven by Olig2 and Brg1, and Chd7 can complex with Sox10 to bind and activate the transcription of genes promoting OPC differentiation ( $\mathrm{He}$ et al., 2016). Interestingly, in OPCs, Chd7 is required for chromatin opening and gene transcription of Sox10 (Marie et al., 2018), suggesting that Chd7 remodeling activity leads to Sox10 upregulation, which in turn promotes OPC differentiation (Figure 2). In OPCs, Chd8 mostly binds to the promoter regions of genes also enriched in Olig2 and Sox10 binding, including histone methyltransferase KMT2/MLL target genes and H3K4me3-marked genes, which are downregulated in Chd8-deficient cells. Moreover, given that KMT2/MLL catalyzes the H3K4me3 mark of promoter activity and Chd8 forms a complex with the KMT2 components, ASH2L and WDR5, this suggests that Chd8 promotes a transcriptional activation program for OPC differentiation (Figure 2). Altogether, these studies indicate that Brg1, Chd8 and Chd7 are coexpressed in OPCs, bind and regulate each other's expression and contribute to different OPC cellfates, with partially redundant functions during developmental myelination (Figure 2), and at least Chd7 \& Chd8 during adult remyelination (Figure 3). Interestingly, Brg1 binds to different genes involved in OPC differentiation compared to those largely co-bound by Chd7 and Chd8 (Marie et al., 2018), suggesting that they could regulate different genetic programs involved in OPC differentiation. Whether the loss of Chd7 and Chd8 function together blocks OPC differentiation or if Brg1 alone is sufficient to promote this process in certain contexts, awaits future investigation.

\section{Perspectives}

\subsection{Limitations of the present chromatin binding profile datasets}

The understanding of chromatin remodeling factors in oligodendrogenesis has made a long step forward with the knowledge acquired in the last years. However, there are still some caveats, and controversial results, in these studies that need to be solved. On the one hand, the profiling of chromatin binding by different factors has been mostly performed from OPCs obtained from neonatal rat primary cultures, and only in few cases, from in vivo OPCs obtained directly from mouse cortices, thus it will be important to show that the chromatin binding of all these factors is independent of either the species of origin or the in vivo versus in vitro source of OPCs, and conserved in human OPCs, allowing future therapeutic developments. On the other hand, many of these datasets need replication to show the robustness of binding in different OPC/OL contexts, including possible developmental stage, regional, sex and species differences. 


\subsection{Interaction between SWI/SNF/BAF and CHD remodeling factors}

An important remaining question is whether different remodeling complexes interact with each other during oligodendrogenesis. For instance, Chd8 was identified as a Chd7 binding partner using a yeast two-hybrid library screen (Batsukh et al., 2010). Furthermore, inducing a Chd7 missense mutation found in CHARGE syndrome patients, caused a disruption of the Chd7-Chd8 interaction in a yeast twohybrid experiment but was not observed in co-immunoprecipitation experiments, suggesting that this interaction could be both direct and indirect (Batsukh et al., 2010). The role of a Chd7-Chd8 protein interaction in oligodendrogenesis awaits further studies. Also, proteomic analysis of Chd7 in human neural crest-like cells showed that Chd7 associates with chromatin remodeling complexes which contain Brg1 (Bajpai et al., 2010). However, while comparison of Chd7 (Schnetz et al., 2009) and Brg1 (Lena Ho et al., 2009) binding sites in mouse ESCs showed that most of these regions are commonly bound by both remodelers (Bajpai et al., 2010), on the contrary, in OPCs, Brg1 shows little overlap with the gene targets of Chd7 and Chd8 (Marie et al., 2018). Thus, understanding how Brg1 and CHD remodelers are brought to different regulatory regions in order to control the various aspects of oligodendrocyte differentiation will help us to fully understand how to boost this process in the context of remyelination.

\subsection{Activator or repressor functions of Chd7/8}

Most studies providing chromatin state of Chd7 mutant cells seem to suggest that Chd7 works as a chromatin opener and thus, transcriptional activator. In this sense, accessible chromatin profiling on Chd7iKO NSCs showed that Chd7 deletion leads to more compacted chromatin in Sox4 and Sox11 promoters, two targets of Chd7 in NSCs (Feng et al., 2013). Furthermore, binding and chromatin accessible profiling in Chd7iKO granule neuron progenitors showed that Chd7 participates in keeping an open chromatin state in genes involved in neuronal differentiation like Reln (Feng et al., 2017; Whittaker et al., 2017). However, in Chd7iKO OPCs, many Chd7-bound genes are upregulated suggesting a direct role of Chd7 in chromatin closing and gene repression, as demonstrated for Trp53/p53 locus (Marie et al., 2018). Furthermore, Chd8 acts as an activator (Durak et al., 2016; Rodriguez-Paredes, Ceballos-Chavez, Esteller, Garcia-Dominguez, \& Reyes, 2009) but also as a repressor of gene expression (Nishiyama et al., 2009; Sakamoto et al., 2000; Yates, Menon, Thompson, \& Bochar, 2010), depending of the cell types. Therefore, how Chd7 and Chd8 can lead to either transcription activation or repression depending on the target gene or cellular context needs further investigation to understand molecular nature of these different contexts.

\subsection{Further direction and unanswered questions}


Despite recent progress, how chromatin remodelers function to modulate an active chromatin state in a cell-type specific manner during OL lineage progression and myelin repair remains unknown. Currently, we still have an incomplete understanding of the contributions of chromatin remodeling factors at each stage of $\mathrm{OL}$ during development and regeneration after injury. For example, it remains to be determined whether Chd7 and Chd8, mutations of which lead to severe developmental disorders in humans, are involved in OPC specification from NSCs. The roles of other seven CHD family members in oligodendrogenesis have not yet studied as well. Through recent efforts, we are at the earliest stages of beginning to understand the role of chromatin-remodeling complexes during $\mathrm{OL}$ lineage development, but we have yet to obtain a clear picture of how these mechanisms are regulated by TFs or by environmental signaling or inputs to carry out this process. Additionally, as chromatin dynamics are considered long-range interactions in 3D space, how these chromatin remodelers act to regulate 3D genomic folding at each stage of OL development remains to be defined.

Given the strong requirements for these chromatin remodelers in mediating OL lineage development, they may have important implications for a better understanding of normal development and the treatment of human disease. Due to the lack of specific small-molecule modulators, the value of chromatin remodelers has yet to be fully explored as therapeutic targets for promoting OPC proliferation and differentiation in neurological diseases such as multiple sclerosis and Alzheimer's disease, where defects in the myelin repair process have been identified. Future exploratory studies into the regulation of OL biology in these complex human diseases will reveal how chromatin remodeling is regulated under pathological conditions and suggest potential therapies or interventions. 


\section{Figure legends:}

Figure 1. Expression profile of chromatin remodelers and transcription factors during oligodendrogenesis.

A. Protein expression levels of chromatin remodelers and transcription factors in NSCs/NPCs, OPCs and immature and myelinating OLs. B. Comparison of the RNA expression levels of chromatin remodelers and transcription factors from different database: (a) Bulk RNA-seq of different purified cell-types from P7 (neurons, astrocytes and microglia) and P17 (OPCs, iOLs and mOLs) mouse brain (Y. Zhang et al., 2014); (b) single cell RNA-seq (scRNA-seq) of non-neuronal isolated cell populations from the adult mouse dentate gyrus (Artegiani et al., 2017); (c) scRNA-seq done in E13.5, P7, and juvenile and adult mice mouse central nervous system describing the early stages of the OL lineage (Marques et al., 2018); (d) scRNA-seq done in juvenile and adult mouse central nervous system, covering 10 different regions and describing gene expression in the OL lineage (Marques et al., 2016). Intensity of color represent the relative expression level of each gene. Neural stem cells (NSC), neural progenitor cells (NPC and NP), primitive OPCs (pri-OPC), cycling OPC (OPCcyc), immature oligodendrocyte (iOL) containing committed OPC (COP) and newly-formed OL (NFOL), mature OL (mOL) containing myelinforming $\mathrm{OL}(\mathrm{MFOL})$ and myelinating $\mathrm{OL}(\mathrm{MOL})$, neuron $(\mathrm{N})$ containing interneuron (IN), astrocytes (A) and Microglia (M).

Figure 2. Model of regulation of oligodendrogenesis by chromatin remodelers and TFs.

In red, factors function in each process. Activation is depictured by a positive circle and inhibition by a negative circle. Blue ellipses represent the cell nuclei highlighting the factors involved in each step of oligodendrogenesis. Question marks indicate hypothetical function still not addressed. NSC, neural stem cell; OPC, oligodendrocyte precursor cell; OL, oligodendrocyte.

Figure 3: Model of regulation of remyelination by chromatin remodelers and TFs.

In red, factors function in each process. Activation is depictured by a positive circle and inhibition by a negative circle. Blue ellipses represent the cell nuclei highlighting the factors involved in $\mathrm{OL}$ differentiation and OPC proliferation. NSC, neural stem cell; OPC, oligodendrocyte precursor cell; OL, oligodendrocyte.

Figure 4: Model of cooperation between chromatin remodelers and transcription factors for the transcription activation of OPC differentiation genes. (1) Olig2 binds first as a pioneer TF and (2) recruits chromatin remodelers $\mathrm{Chd7}$ and $\mathrm{Chd8}$, which (3) remove nucleosomes, making the chromatin accessible for the binding of Sox10. (4) All together, chromatin remodelers and TFs participate in the recruitment of the transcription initiation complex and allow a robust gene expression. (5) The removal of chromatin remodelers marks the downregulation. Adapted from (Marie et al., 2018). 
Table 1. Summary of chromatin remodelers requirement and binding in oligodendrogenesis

\begin{tabular}{|c|c|c|c|c|}
\hline & Brg1 & Brm & Chd7 & Chd8 \\
\hline $\begin{array}{l}\text { OPC } \\
\text { specification }\end{array}$ & $\begin{array}{l}\text { Strong reduction in OPCs (Nestin- } \\
\text { Cre or Brn4-Cre). Olig2+ cells but } \\
\text { not OPC traits. Caveat: other } \\
\text { changes in NSC biology and diff } \\
\text { of all neural lineages. No changes } \\
\text { spinal cord (Olig1-Cre) }\end{array}$ & N/A & N/A & N/A \\
\hline $\begin{array}{l}\text { OPC } \\
\text { differentiation }\end{array}$ & $\begin{array}{l}\text { Strong }(>90 \%) \text { reduction (Olig1- } \\
\text { Cre) or partial }(40 \%) \text { reduction } \\
(C N P-C r e \text { or NG2-Cre). }\end{array}$ & $\begin{array}{l}\text { No change } \\
\text { (shRNA in vitro) }\end{array}$ & $\begin{array}{l}\text { Reduced numbers of } \\
\text { differentiating OPCs }\end{array}$ & $\begin{array}{l}\text { Blocked in spinal cord \& } \\
\text { cerebellum, and reduced in } \\
\text { the forebrain (Olig1-Cre); } \\
\text { Strong ( } 85 \% \text { ) reduction in } \\
\text { brain \& spinal cord (PDGFRa- } \\
\text { CreERT). Reduced ( } 36 \% \text { ) OL } \\
\text { numbers in Chd8 knockdown } \\
\text { in culture (shRNA) }\end{array}$ \\
\hline $\begin{array}{l}\text { OPC } \\
\text { proliferation }\end{array}$ & No changes (Olig1-Cre) & N/A & $\begin{array}{l}\text { No change postnatal brain } \\
\text { (PDGFRa-CreERT). No } \\
\text { change in brain \& spinal } \\
\text { cord (Olig1-Cre). Reduced } \\
\text { in spinal cord (PDGFRa- } \\
\text { CreERT). }\end{array}$ & $\begin{array}{l}\text { Strong }(90 \%) \text { reduced } \\
\text { numbers and proliferation of } \\
\text { OPCs in spinal cord but not } \\
\text { in the forebrain (Olig1-Cre). } \\
\text { No changes in numbers and } \\
\text { proliferation of OPCs in } \\
\text { forebrain \& spinal cord } \\
\text { (PDGFRa-CreERT). }\end{array}$ \\
\hline OPC survival & N/A & N/A & $\begin{array}{l}\text { p53-mediated apoptosis of } \\
\text { non-cycling OPCs } \\
\text { (PDGFRa-CreERT). }\end{array}$ & $\begin{array}{l}\text { Apoptosis of OPCs (PDGFRa- } \\
\text { CreERT). }\end{array}$ \\
\hline Myelination & $\begin{array}{l}\text { Strong reduction/absence } \\
\text { (Olig1-Cre) }\end{array}$ & $\begin{array}{l}\text { No change } \\
\text { (shRNA in vitro) }\end{array}$ & $\begin{array}{l}\text { partial reduction but } \\
\text { compensated with time } \\
(P D G F R a-C r e E R T)\end{array}$ & $\begin{array}{l}\text { Strong reduction/absence } \\
\text { (Olig1-Cre) }\end{array}$ \\
\hline Remyelination & N/A & N/A & $\begin{array}{l}\text { Small reduction but } \\
\text { compensated with time } \\
\text { (Olig1-Cre) }\end{array}$ & $\begin{array}{l}\text { Strong (12-fold) reduction } \\
\text { (PDGFRa-CreERT) }\end{array}$ \\
\hline TF interaction & Olig2 & $?$ & Sox10 & N/A \\
\hline ChIP-seq data & $\begin{array}{l}\text { - OPCs: rat ( } 400 \text { peaks, } \mathrm{P} 1 \\
\text { primary cultures) } \\
\text { Failed ChIP? } \\
\text { - OLs: rat (5,000 peaks, P1 } \\
\text { primary cultures) }\end{array}$ & N/A & $\begin{array}{l}\text { - OPCs: mouse }(11,000 \\
\text { peaks, in vivo } \mathrm{P} 7) \\
\text { - OLs: rat (15,000 peaks, } \\
\text { P1 primary cultures) }\end{array}$ & $\begin{array}{l}\text { - OPCs: mouse }(18,000 \\
\text { peaks, in vivo P7) \& rat } \\
\text { (23,000 peaks, P1 } \\
\text { primary cultures) } \\
\text { - OLs: rat ( } 11,000 \text { peaks, } \\
\text { P1 primary cultures) }\end{array}$ \\
\hline
\end{tabular}

\section{Conflict of interest}

Authors have not conflict of interest.

\section{Acknowledgments}

This work was supported by funding by grants from the National Multiple Sclerosis Society (NMSS RG-1501-02851), and the Fondation pour l'Aide à la Recherche sur la Sclérose en Plaques (ARSEP, 2014, 2015, 2018, 2019). C.M. was supported by funding from Sorbonne Universités and Fondation pour la Recherche Médicale (FRM), NMSS and ARSEP. Q.R.L. is supported by grants from the US National Institutes of Health R01NS072427 and R01NS075243. 


\section{References:}

Akazawa, C., Sasai, Y., Nakanishi, S., \& Kageyama, R. (1992). Molecular characterization of a rat negative regulator with a basic helix-loop-helix structure predominantly expressed in the developing nervous system. Journal of Biological Chemistry, 267(30), 21879-21885.

Alvarez-Saavedra, M., De Repentigny, Y., Lagali, P. S., Raghu Ram, E. V., Yan, K., Hashem, E., . . Picketts, D. J. (2014). Snf2h-mediated chromatin organization and histone H1 dynamics govern cerebellar morphogenesis and neural maturation. Nat Commun, 5, 4181. doi:10.1038/ncomms5181

Artegiani, B., Lyubimova, A., Muraro, M., van Es, J. H., van Oudenaarden, A., \& Clevers, H. (2017). A Single-Cell RNA Sequencing Study Reveals Cellular and Molecular Dynamics of the Hippocampal Neurogenic Niche. Cell Reports, 21(11), 3271-3284. doi:10.1016/j.celrep.2017.11.050

Bajpai, R., Chen, D. A., Rada-Iglesias, A., Zhang, J., Xiong, Y., Helms, J., . . Wysocka, J. (2010). CHD7 cooperates with PBAF to control multipotent neural crest formation. Nature, 463(7283), 958962. doi:nature08733 [pii]10.1038/nature08733

Barski, A., Cuddapah, S., Cui, K., Roh, T.-Y., Schones, D. E., Wang, Z., .. Z Zhao, K. (2007). High-Resolution Profiling of Histone Methylations in the Human Genome. Cell, 129(4), 823-837. doi:10.1016/j.cell.2007.05.009

Batsukh, T., Pieper, L., Koszucka, A. M., von Velsen, N., Hoyer-Fender, S., Elbracht, M., . . Pauli, S. (2010). CHD8 interacts with CHD7, a protein which is mutated in CHARGE syndrome. Human Molecular Genetics, 19(14), 2858-2866. doi:10.1093/hmg/ddq189

Benezra, R., Davis, R. L., Lockshon, D., Turner, D. L., \& Weintraub, H. (1990). The protein Id: A negative regulator of helix-loop-helix DNA binding proteins. Cell, 61(1), 49-59.

Bertrand, N., Castro, D. S., \& Guillemot, F. (2002). Proneural genes and the specification of neural cell types. Nat Rev Neurosci, 3(7), 517-530.

Bischof, M., Weider, M., Küspert, M., Nave, K.-A., \& Wegner, M. (2015). Brg1-Dependent Chromatin Remodelling Is Not Essentially Required during Oligodendroglial Differentiation. The Journal of Neuroscience, 35(1), 21-35. doi:10.1523/jneurosci.1468-14.2015

Cai, J., Qi, Y., Hu, X., Tan, M., Liu, Z., Zhang, J., . . . Qiu, M. (2005). Generation of Oligodendrocyte Precursor Cells from Mouse Dorsal Spinal Cord Independent of Nkx6 Regulation and Shh Signaling. Neuron, 45(1), 41-53.

Cai, Y., Brophy, P. D., Levitan, I., Stifani, S., \& Dressler, G. R. (2003). Groucho suppresses Pax2 transactivation by inhibition of JNK-mediated phosphorylation. The EMBO Journal, 22(20), 5522-5529. doi:10.1093/emboj/cdg536

Chen, G., Fernandez, J., Mische, S., \& Courey, A. J. (1999). A functional interaction between the histone deacetylase $\mathrm{Rpd} 3$ and the corepressor Groucho in Drosophila development. Genes \& Development, 13(17), 2218-2230.

Cirillo, L. A., Lin, F. R., Cuesta, I., Friedman, D., Jarnik, M., \& Zaret, K. S. (2002). Opening of compacted chromatin by early developmental transcription factors HNF3 (FoxA) and GATA-4. Mol Cell, 9(2), 279-289. doi:10.1016/s1097-2765(02)00459-8

Cirillo, L. A., McPherson, C. E., Bossard, P., Stevens, K., Cherian, S., Shim, E. Y., . . Zaret, K. S. (1998). Binding of the winged-helix transcription factor HNF3 to a linker histone site on the nucleosome. Embo J, 17(1), 244-254. doi:10.1093/emboj/17.1.244

Creyghton, M. P., Cheng, A. W., Welstead, G. G., Kooistra, T., Carey, B. W., Steine, E. J., . . Jaenisch, R. (2010). Histone H3K27ac separates active from poised enhancers and predicts developmental state. Proceedings of the National Academy of Sciences, 107(50), 21931-21936. doi:10.1073/pnas.1016071107

Dawson, M. R., Polito, A., Levine, J. M., \& Reynolds, R. (2003). NG2-expressing glial progenitor cells: an abundant and widespread population of cycling cells in the adult rat CNS. Mol Cell Neurosci, 24(2), 476-488. 
Deng, W., Rupon, J. W., Krivega, I., Breda, L., Motta, I., Jahn, K. S., . . Blobel, G. A. (2014). Reactivation of developmentally silenced globin genes by forced chromatin looping. Cell, 158(4), 849-860. doi:10.1016/j.cell.2014.05.050

Doi, T., Ogata, T., Yamauchi, J., Sawada, Y., Tanaka, S., \& Nagao, M. (2017). Chd7 Collaborates with Sox2 to Regulate Activation of Oligodendrocyte Precursor Cells after Spinal Cord Injury. The Journal of Neuroscience, 37(43), 10290-10309. doi:10.1523/jneurosci.1109-17.2017

Durak, O., Gao, F., Kaeser-Woo, Y. J., Rueda, R., Martorell, A. J., Nott, A., . . Tsai, L. H. (2016). Chd8 mediates cortical neurogenesis via transcriptional regulation of cell cycle and Wnt signaling. Nat Neurosci, 19(11), 1477-1488. doi:10.1038/nn.4400

Elsesser, O., Fröb, F., Küspert, M., Tamm, E. R., Fujii, T., Fukunaga, R., \& Wegner, M. (2019). Chromatin remodeler Ep400 ensures oligodendrocyte survival and is required for myelination in the vertebrate central nervous system. Nucleic Acids Research, 47(12), 6208-6224. doi:10.1093/nar/gkz376

Emery, B., Agalliu, D., Cahoy, J. D., Watkins, T. A., Dugas, J. C., Mulinyawe, S. B., . . Barres, B. A. (2009). Myelin gene regulatory factor is a critical transcriptional regulator required for CNS myelination. Cell, 138(1), 172-185.

Feng, W., Kawauchi, D., Körkel-Qu, H., Deng, H., Serger, E., Sieber, L., . . Liu, H.-K. (2017). Chd7 is indispensable for mammalian brain development through activation of a neuronal differentiation programme. Nat Commun, 8, 14758. doi:10.1038/ncomms14758

Feng, W., Khan, Muhammad A., Bellvis, P., Zhu, Z., Bernhardt, O., Herold-Mende, C., \& Liu, H.-K. (2013). The Chromatin Remodeler CHD7 Regulates Adult Neurogenesis via Activation of SoxC Transcription Factors. Cell Stem Cell, 13(1), 62-72.

Ferri, E., Petosa, C., \& McKenna, C. E. (2016). Bromodomains: Structure, function and pharmacology of inhibition. Biochem Pharmacol, 106, 1-18. doi:10.1016/j.bcp.2015.12.005

Filbin, M., \& Monje, M. (2019). Developmental origins and emerging therapeutic opportunities for childhood cancer. Nature Medicine, 25(3), 367-376. doi:10.1038/s41591-019-0383-9

García-Marqués, J., Núñez-Llaves, R., \& López-Mascaraque, L. (2014). NG2-Glia from Pallial Progenitors Produce the Largest Clonal Clusters of the Brain: Time Frame of Clonal Generation in Cortex and Olfactory Bulb. The Journal of Neuroscience, 34(6), 2305-2313. doi:10.1523/jneurosci.3060-13.2014

Guenther, M. G., Levine, S. S., Boyer, L. A., Jaenisch, R., \& Young, R. A. (2007). A Chromatin Landmark and Transcription Initiation at Most Promoters in Human Cells. Cell, 130(1), 77-88. doi:10.1016/j.cell.2007.05.042

Hargreaves, D. C., \& Crabtree, G. R. (2011). ATP-dependent chromatin remodeling: genetics, genomics and mechanisms. Cell Res, 21(3), 396-420. doi:10.1038/cr.2011.32

He, D., Marie, C., Zhao, C., Kim, B., Wang, J., Deng, Y., ... Lu, Q. R. (2016). Chd7 cooperates with Sox10 and regulates the onset of CNS myelination and remyelination. Nat Neurosci, advance online publication. doi:10.1038/nn.4258

Ho, L., \& Crabtree, G. R. (2010). Chromatin remodelling during development. Nature, 463(7280), 474484. doi:nature08911 [pii]10.1038/nature08911

Ho, L., Jothi, R., Ronan, J. L., Cui, K., Zhao, K., \& Crabtree, G. R. (2009). An embryonic stem cell chromatin remodeling complex, esBAF, is an essential component of the core pluripotency transcriptional network. Proceedings of the National Academy of Sciences, 106(13), 5187-5191. doi:10.1073/pnas.0812888106

Jin, Q., Yu, L.-R., Wang, L., Zhang, Z., Kasper, L. H., Lee, J.-E., . . G Ge, K. (2011). Distinct roles of GCN5/PCAF-mediated H3K9ac and CBP/p300-mediated H3K18/27ac in nuclear receptor transactivation. The EMBO Journal, 30(2), 249-262. doi:10.1038/emboj.2010.318

Kageyama, R., Ohtsuka, T., \& Kobayashi, T. (2007). The Hes gene family: repressors and oscillators that orchestrate embryogenesis. Development, 134(7), 1243-1251. doi:10.1242/dev.000786 
Kastenhuber, E. R., \& Lowe, S. W. (2017). Putting p53 in Context. Cell, 170(6), 1062-1078. doi:https://doi.org/10.1016/j.cell.2017.08.028

Kim, T.-K., \& Shiekhattar, R. (2015). Architectural and Functional Commonalities between Enhancers and Promoters. Cell, 162(5), 948-959. doi:10.1016/j.cell.2015.08.008

Kondo, T., \& Raff, M. (2000). The Id4 HLH protein and the timing of oligodendrocyte differentiation. Embo J, 19(9), 1998-2007.

Kuspert, M., Hammer, A., Bosl, M. R., \& Wegner, M. (2011). Olig2 regulates Sox10 expression in oligodendrocyte precursors through an evolutionary conserved distal enhancer. Nucleic Acids Res, 39(4), 1280-1293. doi:gkq951 [pii]10.1093/nar/gkq951

Lang, J., Maeda, Y., Bannerman, P., Xu, J., Horiuchi, M., Pleasure, D., \& Guo, F. (2013). Adenomatous Polyposis Coli Regulates Oligodendroglial Development. The Journal of Neuroscience, 33(7), 3113-3130. doi:10.1523/jneurosci.3467-12.2013

Lawrence, M., Daujat, S., \& Schneider, R. (2016). Lateral Thinking: How Histone Modifications Regulate Gene Expression. Trends in Genetics, 32(1), 42-56. doi:https://doi.org/10.1016/j.tig.2015.10.007

Liu, A., Li, J., Marin-Husstege, M., Kageyama, R., Fan, Y., Gelinas, C., \& Casaccia-Bonnefil, P. (2006). A molecular insight of Hes5-dependent inhibition of myelin gene expression: old partners and new players. Embo J, 25(20), 4833-4842.

Liu, C., Sage, Jonathan C., Miller, Michael R., Verhaak, Roel G. W., Hippenmeyer, S., Vogel, H., .. Z Zong, H. (2011). Mosaic Analysis with Double Markers Reveals Tumor Cell of Origin in Glioma. Cell, 146(2), 209-221.

Liu, Z., Hu, X., Cai, J., Liu, B., Peng, X., Wegner, M., \& Qiu, M. (2007). Induction of oligodendrocyte differentiation by Olig2 and Sox10: evidence for reciprocal interactions and dosage-dependent mechanisms. Dev Biol, 302(2), 683-693.

Lu, Q. R., Sun, T., Zhu, Z., Ma, N., Garcia, M., Stiles, C. D., \& Rowitch, D. H. (2002). Common developmental requirement for Olig function indicates a motor neuron/oligodendrocyte connection. Cell, 109(1), 75-86.

Lu, Q. R., Yuk, D., Alberta, J. A., Zhu, Z., Pawlitzky, I., Chan, J., . . Rowitch, D. H. (2000). Sonic hedgehog-regulated oligodendrocyte lineage genes encoding bHLH proteins in the mammalian central nervous system. Neuron, 25(2), 317-329.

Malarkey, C. S., \& Churchill, M. E. (2012). The high mobility group box: the ultimate utility player of a cell. Trends Biochem Sci, 37(12), 553-562. doi:10.1016/j.tibs.2012.09.003

Marie, C., Clavairoly, A., Frah, M., Hmidan, H., Yan, J., Zhao, C., . . Parras, C. (2018). Oligodendrocyte precursor survival and differentiation requires chromatin remodeling by Chd7 and Chd8. Proceedings of the National Academy of Sciences, 115 (35). doi:10.1073/pnas.1802620115

Marques, S., van Bruggen, D., Vanichkina, D. P., Floriddia, E. M., Munguba, H., Väremo, L., . . CasteloBranco, G. (2018). Transcriptional Convergence of Oligodendrocyte Lineage Progenitors during Development. Developmental Cell, 46(4), 504-517.e507. doi:10.1016/j.devcel.2018.07.005

Marques, S., Zeisel, A., Codeluppi, S., van Bruggen, D., Mendanha Falcão, A., Xiao, L., . . CasteloBranco, G. (2016). Oligodendrocyte heterogeneity in the mouse juvenile and adult central nervous system. Science, 352(6291), 1326-1329. doi:10.1126/science.aaf6463

Marshall, C. A. G., Novitch, B. G., \& Goldman, J. E. (2005). Olig2 Directs Astrocyte and Oligodendrocyte Formation in Postnatal Subventricular Zone Cells. J. Neurosci., 25(32), 7289-7298.

Matsumoto, S., Banine, F., Feistel, K., Foster, S., Xing, R., Struve, J., \& Sherman, L. S. (2016). Brg1 directly regulates Olig2 transcription and is required for oligodendrocyte progenitor cell specification. Developmental Biology, 413(2), 173-187. doi:https://doi.org/10.1016/j.ydbio.2016.04.003

Matsumoto, S., Banine, F., Struve, J., Xing, R., Adams, C., Liu, Y., . . Sherman, L. S. (2006). Brg1 is required for murine neural stem cell maintenance and gliogenesis. Developmental Biology, 289(2), 372-383.

Micucci, J. A., Layman, W. S., Hurd, E. A., Sperry, E. D., Frank, S. F., Durham, M. A., . . Martin, D. M. (2014). CHD7 and retinoic acid signaling cooperate to regulate neural stem cell and inner ear 
development in mouse models of CHARGE syndrome. Human Molecular Genetics, 23(2), 434448. doi:10.1093/hmg/ddt435

Nakatani, H., Martin, E., Hassani, H., Clavairoly, A., Maire, C. L., Viadieu, A., . . Parras, C. (2013). Ascl1/Mash1 Promotes Brain Oligodendrogenesis during Myelination and Remyelination. J Neurosci, 33(23), 9752-9768. doi:10.1523/JNEUROSCI.0805-13.2013

Nielsen, J. A., Hudson, L. D., \& Armstrong, R. C. (2002). Nuclear organization in differentiating oligodendrocytes. Journal of Cell Science, 115(21), 4071-4079. doi:10.1242/jcs.00103

Nishiyama, M., Nakayama, K., Tsunematsu, R., Tsukiyama, T., Kikuchi, A., \& Nakayama, K. I. (2004). Early Embryonic Death in Mice Lacking the $\beta$-Catenin-Binding Protein Duplin. Molecular and Cellular Biology, 24(19), 8386-8394. doi:10.1128/mcb.24.19.8386-8394.2004

Nishiyama, M., Oshikawa, K., Tsukada, Y.-i., Nakagawa, T., lemura, S.-i., Natsume, T., . . Nakayama, K. I. (2009). CHD8 suppresses p53-mediated apoptosis through histone H1 recruitment during early embryogenesis. Nat Cell Biol, 11(2), 172-182.

Norton, J. D., Deed, R. W., Craggs, G., \& Sablitzky, F. (1998). Id helix-loop-helix proteins in cell growth and differentiation. Trends Cell Biol, 8(2), 58-65.

Novitch, B. G., Chen, A. I., \& Jessell, T. M. (2001). Coordinate regulation of motor neuron subtype identity and pan-neuronal properties by the bHLH repressor Olig2. Neuron, 31(5), 773-789.

Panne, D. (2008). The enhanceosome. Curr Opin Struct Biol, 18(2), 236-242. doi:10.1016/j.sbi.2007.12.002

Rada-Iglesias, A., Bajpai, R., Swigut, T., Brugmann, S. A., Flynn, R. A., \& Wysocka, J. (2011). A unique chromatin signature uncovers early developmental enhancers in humans. Nature, 470(7333), 279-283.

Raposo, Alexandre A. S. F., Vasconcelos, Francisca F., Drechsel, D., Marie, C., Johnston, C., Dolle, D., . . . Castro, Diogo S. (2015). Ascl1 Coordinately Regulates Gene Expression and the Chromatin Landscape during Neurogenesis. Cell Reports, 10(9), 1544-1556.

Rodriguez-Paredes, M., Ceballos-Chavez, M., Esteller, M., Garcia-Dominguez, M., \& Reyes, J. C. (2009). The chromatin remodeling factor CHD8 interacts with elongating RNA polymerase II and controls expression of the cyclin E2 gene. Nucleic Acids Res, 37(8), 2449-2460. doi:10.1093/nar/gkp101

Sakamoto, I., Kishida, S., Fukui, A., Kishida, M., Yamamoto, H., Hino, S.-i., ... Kikuchi, A. (2000). A Novel $\beta$-Catenin-binding Protein Inhibits $\beta$-Catenin-dependent Tcf Activation and Axis Formation. Journal of Biological Chemistry, 275(42), 32871-32878.

Schnetz, M. P., Bartels, C. F., Shastri, K., Balasubramanian, D., Zentner, G. E., Balaji, R., . . Scacheri, P. C. (2009). Genomic distribution of CHD7 on chromatin tracks H3K4 methylation patterns. Genome Research, 19(4), 590-601. doi:10.1101/gr.086983.108

Sekiya, T., \& Zaret, K. S. (2007). Repression by Groucho/TLE/Grg Proteins: Genomic Site Recruitment Generates Compacted Chromatin In Vitro and Impairs Activator Binding In Vivo. Molecular Cell, 28(2), 291-303. doi:10.1016/j.molcel.2007.10.002

Soufi, A., Garcia, Meilin F., Jaroszewicz, A., Osman, N., Pellegrini, M., \& Zaret, Kenneth S. (2015). Pioneer Transcription Factors Target Partial DNA Motifs on Nucleosomes to Initiate Reprogramming. Cell, 161(3), 555-568.

Stolt, C. C., Rehberg, S., Ader, M., Lommes, P., Riethmacher, D., Schachner, M., ... Wegner, M. (2002). Terminal differentiation of myelin-forming oligodendrocytes depends on the transcription factor Sox10. Genes Dev, 16(2), 165-170.

Turnescu, T., Arter, J., Reiprich, S., Tamm, E. R., Waisman, A., \& Wegner, M. (2018). Sox8 and Sox10 jointly maintain myelin gene expression in oligodendrocytes. Glia, 66(2), 279-294. Van Nostrand, J. L., Brady, C. A., Jung, H., Fuentes, D. R., Kozak, M. M., Johnson, T. M., . . Attardi, L. D. (2014). Inappropriate p53 Activation During Development Induces Features of CHARGE Syndrome. Nature, 514(7521), 228-232. doi:10.1038/nature13585

Vogl, M. R., Reiprich, S., Küspert, M., Kosian, T., Schrewe, H., Nave, K.-A., \& Wegner, M. (2013). Sox10 Cooperates with the Mediator Subunit 12 during Terminal Differentiation of Myelinating Glia. The Journal of Neuroscience, 33(15), 6679-6690. doi:10.1523/jneurosci.5178-12.2013 
Wang, S., Sdrulla, A., Johnson, J. E., Yokota, Y., \& Barres, B. A. (2001). A Role for the Helix-Loop-Helix Protein Id2 in the Control of Oligodendrocyte Development. Neuron, 29(3), 603-614.

Wang, Z., Zang, C., Rosenfeld, J. A., Schones, D. E., Barski, A., Cuddapah, S., . . . Zhao, K. (2008). Combinatorial patterns of histone acetylations and methylations in the human genome. Nature Genetics, 40(7), 897-903. doi:10.1038/ng.154

Wapinski, Orly L., Vierbuchen, T., Qu, K., Lee, Qian Y., Chanda, S., Fuentes, Daniel R., . . Wernig, M. (2013). Hierarchical Mechanisms for Direct Reprogramming of Fibroblasts to Neurons. Cell, 155(3), 621-635.

Wegener, A., Deboux, C., Bachelin, C., Frah, M., Kerninon, C., Seilhean, D., . . Nait-Oumesmar, B. (2015). Gain of Olig2 function in oligodendrocyte progenitors promotes remyelination. Brain, 138(1), 120-135. doi:10.1093/brain/awu375

Weider, M., Küspert, M., Bischof, M., Vogl, Michael R., Hornig, J., Loy, K., . . Wegner, M. (2012). Chromatin-Remodeling Factor Brg1 is Required for Schwann Cell Differentiation and Myelination. Developmental Cell, 23(1), 193-201.

Weng, Q., Wang, J., Wang, J., He, D., Cheng, Z., Zhang, F., . . . Lu, Q. R. (2019). Single-Cell Transcriptomics Uncovers Glial Progenitor Diversity and Cell Fate Determinants during Development and Gliomagenesis. Cell Stem Cell, 24(5), 707-723.e708.

Whittaker, D. E., Riegman, K. L., Kasah, S., Mohan, C., Yu, T., Sala, B. P., . . Basson, M. A. (2017). The chromatin remodeling factor CHD7 controls cerebellar development by regulating reelin expression. J Clin Invest, 127(3), 874-887. doi:10.1172/JCI83408

Woodage, T., Basrai, M. A., Baxevanis, A. D., Hieter, P., \& Collins, F. S. (1997). Characterization of the CHD family of proteins. Proceedings of the National Academy of Sciences, 94(21), 1147211477. doi:10.1073/pnas.94.21.11472

Xin, M., Yue, T., Ma, Z., Wu, F.-f., Gow, A., \& Lu, Q. R. (2005). Myelinogenesis and Axonal Recognition by Oligodendrocytes in Brain Are Uncoupled in Olig1-Null Mice. J. Neurosci., 25(6), 1354-1365.

Yates, J. A., Menon, T., Thompson, B. A., \& Bochar, D. A. (2010). Regulation of HOXA2 gene expression by the ATP-dependent chromatin remodeling enzyme CHD8. FEBS Letters, 584(4), 689-693. doi:10.1016/j.febslet.2010.01.022

Ye, F., Chen, Y., Hoang, T., Montgomery, R. L., Zhao, X.-h., Bu, H., . . Lu, Q. R. (2009). HDAC1 and HDAC2 regulate oligodendrocyte differentiation by disrupting the [beta]-catenin-TCF interaction. Nat Neurosci, 12(7), 829-838.

Yen, K., Vinayachandran, V., \& Pugh, B. F. (2013). SWR-C and INO80 chromatin remodelers recognize nucleosome-free regions near +1 nucleosomes. Cell, 154(6), 1246-1256. doi:10.1016/j.cell.2013.08.043

Yip, D. J., Corcoran, C. P., Alvarez-Saavedra, M., DeMaria, A., Rennick, S., Mears, A. J., . . Picketts, D. J. (2012). Snf2I regulates Foxg1-dependent progenitor cell expansion in the developing brain. Dev Cell, 22(4), 871-878. doi:10.1016/j.devcel.2012.01.020

Yu, Y., Chen, Y., Kim, B., Wang, H., Zhao, C., He, X., . . Lu, Q. R. (2013). Olig2 Targets Chromatin Remodelers to Enhancers to Initiate Oligodendrocyte Differentiation. Cell, 152(1-2), 248-261.

Yue, T., Xian, K., Hurlock, E., Xin, M., Kernie, S. G., Parada, L. F., \& Lu, Q. R. (2006). A Critical Role for Dorsal Progenitors in Cortical Myelination 10.1523/JNEUROSCI.4717-05.2006. J. Neurosci., 26(4), 1275-1280.

Zaret, K. S., \& Mango, S. E. (2016). Pioneer transcription factors, chromatin dynamics, and cell fate control. Curr Opin Genet Dev, 37, 76-81. doi:10.1016/j.gde.2015.12.003

Zhang, Y., Chen, K., Sloan, S. A., Bennett, M. L., Scholze, A. R., O'Keeffe, S., ... Wu, J. Q. (2014). An RNASequencing Transcriptome and Splicing Database of Glia, Neurons, and Vascular Cells of the Cerebral Cortex. The Journal of Neuroscience, 34(36), 11929-11947. doi:10.1523/jneurosci.1860-14.2014

Zhao, C., Dong, C., Frah, M., Deng, Y., Marie, C., Zhang, F., . . Lu, Q. R. (2018). Dual Requirement of CHD8 for Chromatin Landscape Establishment and Histone Methyltransferase Recruitment to 
Promote CNS Myelination and Repair. Developmental Cell, ,(6), 753-768.e758. doi:https://doi.org/10.1016/j.devcel.2018.05.022

Zhou, Q., \& Anderson, D. J. (2002). The bHLH transcription factors OLIG2 and OLIG1 couple neuronal and glial subtype specification. Cell, 109(1), 61-73.

Zhou, Q., Wang, S., \& Anderson, D. J. (2000). Identification of a Novel Family of Oligodendrocyte Lineage-Specific Basic Helix-Loop-Helix Transcription Factors. Neuron, 25(2), 331-343. 
A

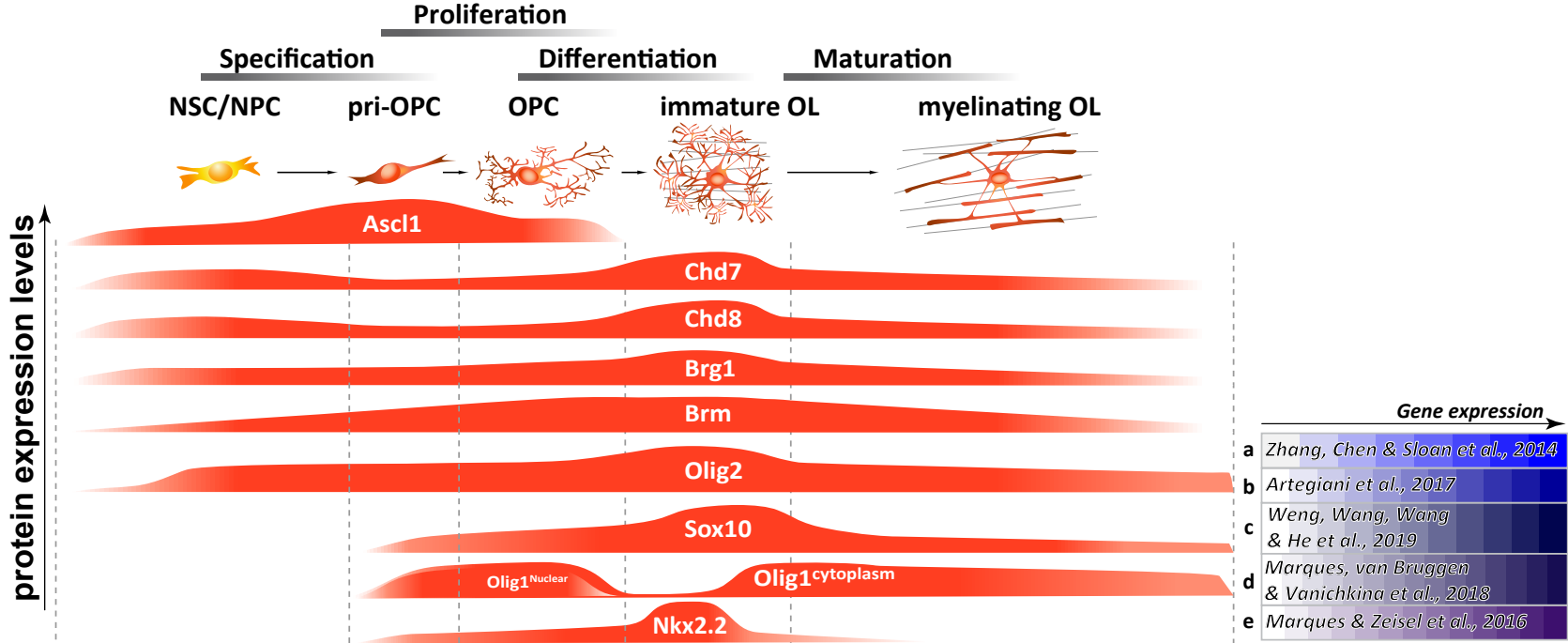

\begin{tabular}{|c|c|c|c|c|c|c|c|c|c|c|c|c|c|c|c|c|}
\hline \multirow{2}{*}{ B } & \multirow{2}{*}{ NSCs } & NPCs & \multirow{2}{*}{ pri-OPCs } & \multicolumn{2}{|r|}{ OPCs } & \multicolumn{2}{|r|}{ iOLs } & \multicolumn{6}{|c|}{ mOLs } & \multirow{2}{*}{$\mathbf{N}$} & \multirow[t]{2}{*}{ A } & \multirow[t]{2}{*}{$\mathbf{M}$} \\
\hline & & \begin{tabular}{|l} 
early NPCs late NPCs \\
NP1a NP1b NP2 NP3
\end{tabular} & & \multicolumn{2}{|r|}{$\begin{array}{l}\text { OPC1b } \\
\text { C1a OPCcyc }\end{array}$} & \multicolumn{2}{|r|}{ NFOL1 } & \multicolumn{6}{|c|}{$\begin{array}{llll}\text { MFOL1 } & \text { MOL1 } & \text { MOL3 } & \text { MOL5 }\end{array}$} & & & \\
\hline \multirow{5}{*}{ Ascl1 $\begin{array}{r}\mathrm{b} \\
\mathrm{c} \\
\mathrm{d} \\
\mathrm{e}\end{array}$} & & & & \multicolumn{2}{|r|}{9.8} & & 1.3 & & & 0.1 & & & & 2.6 & 1.2 & 0.1 \\
\hline & 0.32 & 0.25 & & & 0.55 & 0.10 & & 0.12 & & & & & & 0.10 & & 0.11 \\
\hline & & 1.14 & 4.12 & & 1.68 & & & & & & & & & & & \\
\hline & & $\begin{array}{llll}0.28 & 0.29 & 0.04 & 0.24\end{array}$ & & 0.26 & 0.530 .26 & 0.37 & 0.13 & & & & & & & & & \\
\hline & & & & & 0.4 & 0.15 & 0 & 0 & 0 & 0 & 0 & 0 & 0 & & & \\
\hline & & & & & 8.8 & & 6.0 & & & 1.3 & & & & 5.6 & 8.6 & 3.3 \\
\hline Chd7 & 0.73 & 2.35 & & & 1.23 & 1.19 & & 0.86 & & & & & & 0.10 & & 1.00 \\
\hline Crias & & 2 & 3 & & 6.57 & & & & & & & & & & & \\
\hline & & \begin{tabular}{|l|l|l|l|}
1.91 & 1.21 & 1.52 & 1.14 \\
\end{tabular} & & 1.07 & 1.261 .31 & 1.33 & 1.96 & & & & & & & & & \\
\hline & & & & & 0.9 & 1.1 & $\begin{array}{ll}1.1 & 1.2\end{array}$ & $\begin{array}{ll}1.3 & 1\end{array}$ & 0.9 & 0.8 & 0.7 & 0.6 & 0.6 & & & \\
\hline & & & & & 9.4 & & 7.6 & & & 3.0 & & & & 8.2 & 12.8 & 4.7 \\
\hline Chd8 & 0.48 & 0.92 & & & 0.54 & 0.46 & & 0,39 & & & & & & 0.35 & & 0.49 \\
\hline Crivo & & 0 & 0.31 & & 2.53 & & & & & & & & & & & \\
\hline & & \begin{tabular}{ll|l|l|}
1.45 & 1.29 & 2.07 & 1.14
\end{tabular} & & 0.63 & $0.78 \quad 0,8$ & 1.06 & 1.17 & & & & & & & & & \\
\hline & & & & & 0.5 & 1 & 1 & 1.51 & 0.9 & $1.2 \quad 0.8$ & 0.8 & 0.8 & 0.5 & & & \\
\hline & & & & & 24.8 & & 20.8 & & & 11.8 & & & & 22.7 & 12.9 & 12.3 \\
\hline Smarca4 & 0.40 & 1.16 & & & 0.84 & 0.46 & & 0.59 & & & & & & 0.60 & & 0.54 \\
\hline (Brg1) & & 4.74 & 5.67 & & 11.73 & & & & & & & & & & & \\
\hline & & $\begin{array}{|llll|}1.06 & 1.29 & 3.06 & 1.31 \\
\end{array}$ & & 0.46 & 0.630 .49 & 0.66 & 0.9 & & & & & & & & & \\
\hline & & & & & 0.5 & 0.6 & $0.5 \quad 0.6$ & $0.9 \quad 0.5$ & 0.6 & $0.8 \quad 0.5$ & 0.4 & 0.4 & 0.35 & & & \\
\hline & & & & & 25.1 & & 10.7 & & & 5.6 & & & & 23.3 & 19.8 & 6.5 \\
\hline Smarca2 & 0.62 & 1.16 & & & 0.98 & 0.74 & & 0.56 & & & & & & 3.10 & & 0.70 \\
\hline (Brm) & & 0.23 & 0.23 & & 0.83 & & & & & & & & & & & \\
\hline & & \begin{tabular}{lll|ll}
0.6 & 1.64 & 1.06 & 0.57 \\
\end{tabular} & & 0.47 & $0.8 \quad 0.51$ & 0.45 & 0.5 & & & & & & & & & \\
\hline & & & & & 0.6 & 0.55 & 0.50 .55 & $0.9 \quad 0.7$ & 0.75 & 0.750 .6 & 0.6 & 0 & 0.4 & & & \\
\hline & & & & & 210.2 & & 119.3 & & & 58.6 & & & & 9.5 & 59.4 & 0.4 \\
\hline ? & 0.65 & 0.34 & & & 8.87 & 5.55 & & 2.49 & & & & & & 0.35 & & 0.34 \\
\hline Uilge & & 1.22 & 3.39 & & 2.29 & & & & & & & & & & & \\
\hline & & \begin{tabular}{|l|l|l|l|}
0.45 & 0.07 & 0.05 & 0.27 \\
\end{tabular} & & 1.92 & $1.96 \quad 2.4$ & 2.03 & 2.06 & & & & & & & & & \\
\hline & & & & & 1.2 & 1.4 & $0.8 \quad 1.2$ & $\begin{array}{ll}1.8 & 1 \\
\end{array}$ & 1 & $0.8 \quad 0.5$ & 0.6 & 0.6 & 0.8 & & & \\
\hline & & & & & 147.1 & & 132.6 & & & 103.3 & & & & 3.3 & 0.1 & 0.5 \\
\hline & 0.92 & 0.65 & & & 9.29 & 4.37 & & 5.95 & & & & & & 0.35 & & 0.80 \\
\hline Sox10 & & 0.56 & 0.78 & & 0.88 & & & & & & & & & & & \\
\hline & & \begin{tabular}{|l|l|l|l|}
0.29 & 0 & 0.04 & 0.15 \\
\end{tabular} & & 1.98 & 2.111 .86 & 2.54 & 3.76 & & & & & & & & & \\
\hline & & & & & 1.2 & 1.6 & 1.21 .5 & 1.6 & 1.4 & $\begin{array}{ll}1.4 & 0.8\end{array}$ & 1.2 & 1.2 & 1.5 & & & \\
\hline & & & & & 1360 & & 720.2 & & & 494.5 & & & & 32.0 & 109.5 & 6.2 \\
\hline Olia1 & 0.86 & 0.38 & & & 14.60 & 7.37 & & 6.20 & & & & & & 0.10 & & 0.50 \\
\hline Uing & & 0.92 & 2.35 & & 2.13 & & & & & & & & & & & \\
\hline & & \begin{tabular}{|l|l|l|l|}
0.87 & 0 & 0.24 & 0.68
\end{tabular} & & 4.42 & $5.4 \quad 5.01$ & 4.35 & 3.97 & & & & & & & & & \\
\hline & & & & & 2.5 & 2 & $1.5 \quad 2.5$ & 43 & 2.5 & 2.5 & 2 & 2 & 2 & & & \\
\hline & & & & & 21.8 & & 38.0 & & & 7.7 & & & & 1.1 & 6.8 & 0.1 \\
\hline Nkx2-? & 0.13 & 0.12 & & & 0.73 & 2.10 & & 0.32 & & & & & & 0.10 & & 0.10 \\
\hline $10 \times \times<-2$ & & 1.52 & 1.14 & & 2.43 & & & & & & & & & & & \\
\hline & & \begin{tabular}{|l|l|l|l|}
0.06 & 0 & 0.01 & 0.09 \\
\end{tabular} & & 0.55 & 0.390 .58 & 1.28 & 1.15 & & & & & & & & & \\
\hline & & & & & 0.2 & 0.8 & 0.10 & 0.10 .1 & 0.2 & $0.2 \quad 0.1$ & 0.1 & 0.1 & 0.2 & & & \\
\hline
\end{tabular}




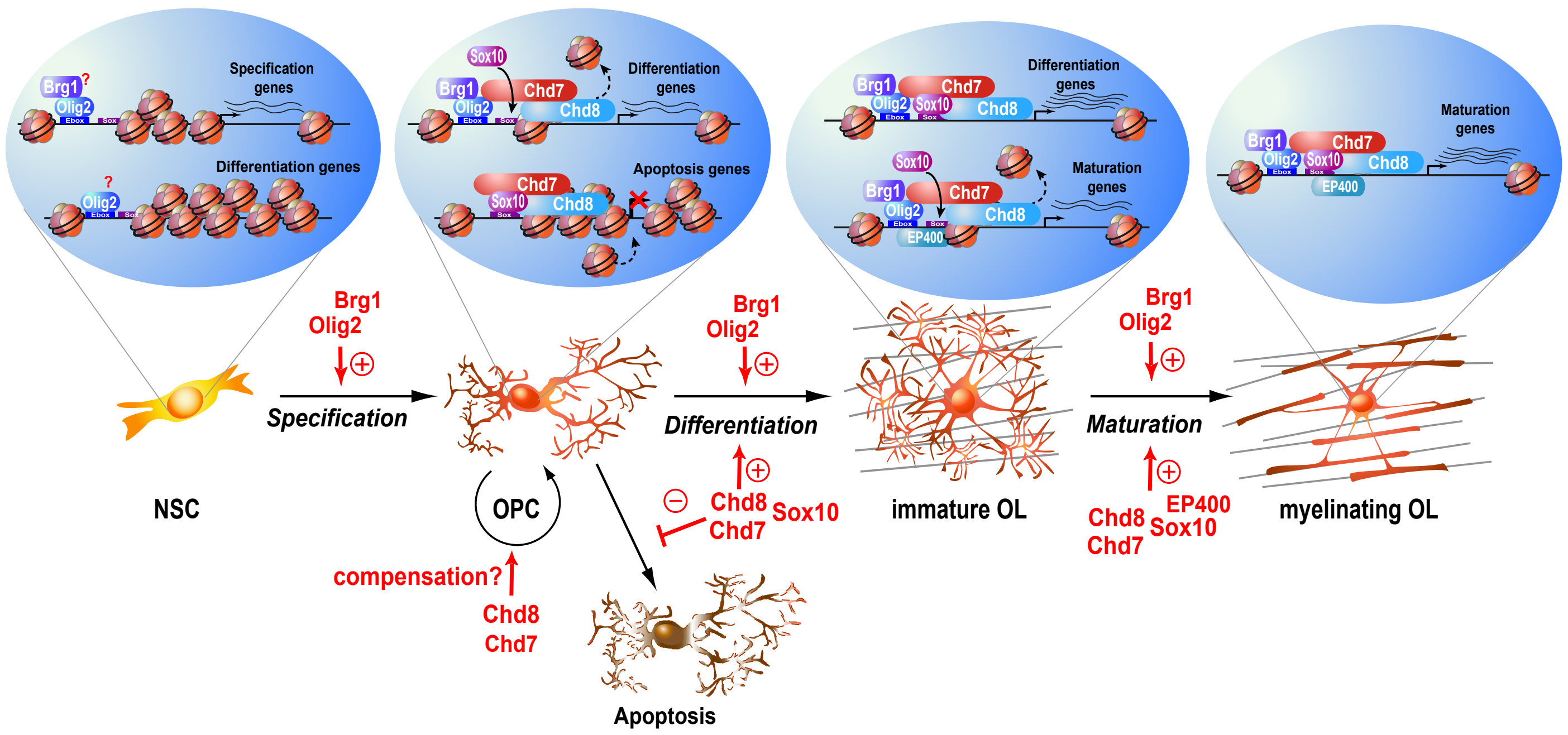


Insult (LPC)

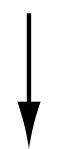

Demyelination

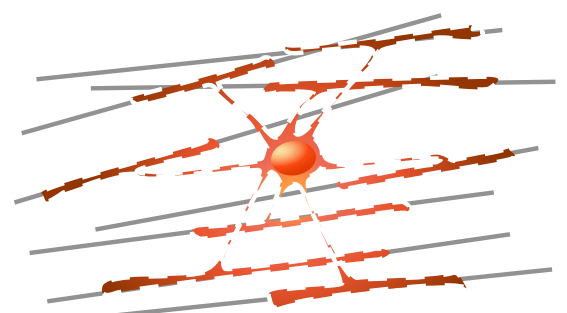

OL death \& myelin loss
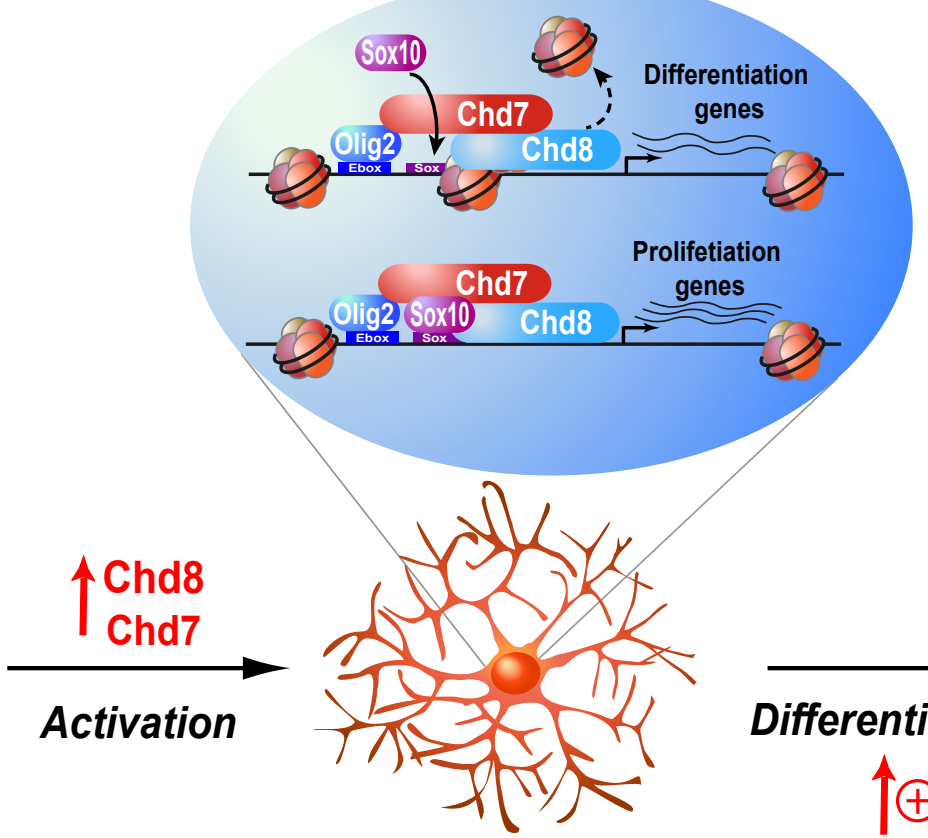
adult OPC

$$
\begin{aligned}
& \text { Chd8 } \\
& \text { Chd7 }
\end{aligned} \underset{\text { Proliferation }}{(}
$$

Differentiation<smiles>[C+]1[CH]CCC1</smiles>

Chd8

Chd7

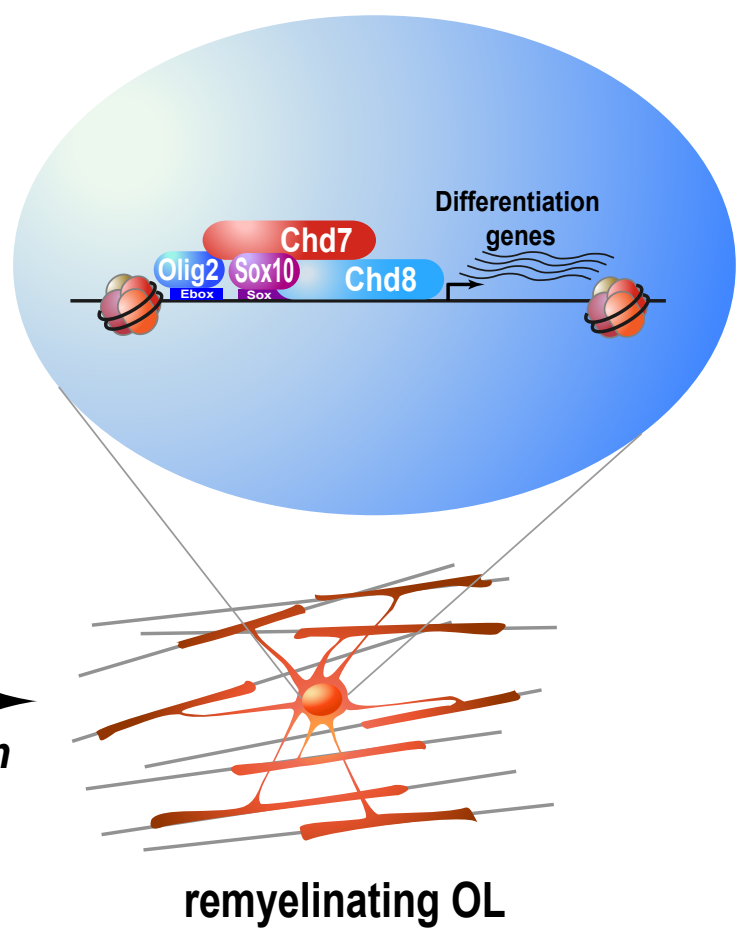


1. Pioneer TFs (Olig2) bind to enhancer in the presence of nucleosomes



2. Recruitement of chromatin remodelers (Chd7 \& Chd8) by pionner TFs

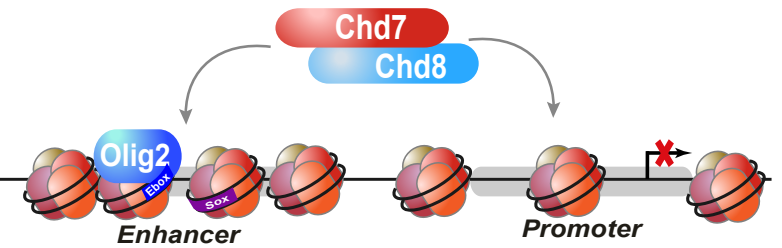

3. Nucleosome release by CHDs \& recruitment of Sox10

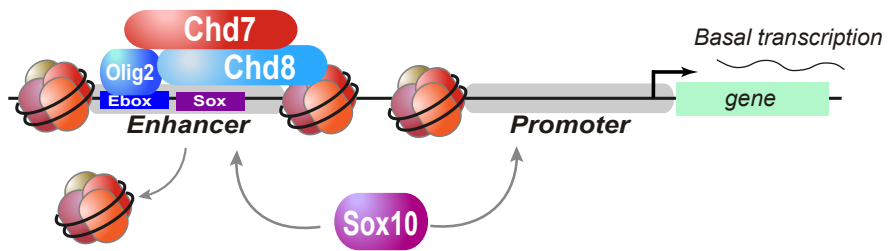

4. Robust gene expression correspond to strong binding of CHDs \& TFs

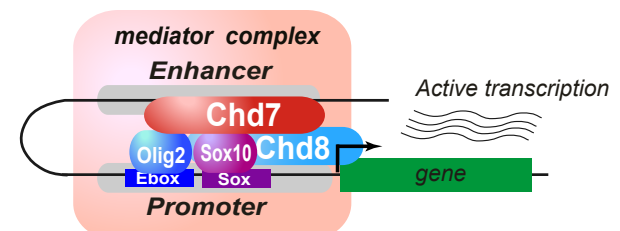

5. Gene downregulation marked by the absence of CHDs

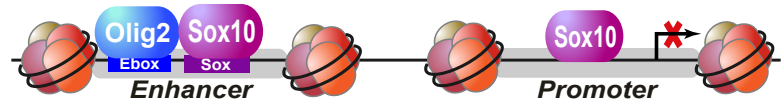

